\title{
A numerical bound for small prime solutions of some ternary linear equations
}

\author{
by \\ Ming-Chit LiU (Hong Kong) and Tianze Wang (Kaifeng)
}

1. Introduction. In this paper, we consider the size of small solutions of the following integral equation (1.1) in prime variables $p_{j}$ :

$$
a_{1} p_{1}+a_{2} p_{2}+a_{3} p_{3}=b .
$$

In particular, we estimate the numerical value of a relevant constant in the upper bound for small prime solutions of (1.1).

Let $a_{1}, a_{2}, a_{3}$ be any integers such that

$$
a_{1} a_{2} a_{3} \neq 0 \quad \text { and } \quad\left(a_{1}, a_{2}, a_{3}\right):=\operatorname{gcd}\left(a_{1}, a_{2}, a_{3}\right)=1 .
$$

Let $b$ be any integer satisfying

$$
b \equiv a_{1}+a_{2}+a_{3}(\bmod 2) \quad \text { and } \quad\left(b, a_{i}, a_{j}\right)=1 \quad \text { for } 1 \leq i<j \leq 3 .
$$

Conditions (1.3) and (1.2) are plainly necessary in our investigation, for otherwise, the equation (1.1) will either be insolvable or be reduced to fewer than three prime variables. The problem on bounds for small prime solutions $p_{1}, p_{2}, p_{3}$ of the equation (1.1) was first considered by A. Baker in connection with his now well-known work [B] on the solvability of certain diophantine inequalities involving primes. Baker's investigation raised immediately the problem of obtaining the best possible upper bound for small prime solutions. As the culmination of a series of earlier discoveries in this context [Li1, Li2], the following was proved [LT1, Theorem 2]:

Theorem 0. Assume the conditions (1.2) and (1.3). If not all $a_{1}, a_{2}, a_{3}$ are of the same sign, then there is an effective absolute constant $B>0$ such that the equation (1.1) has a prime solution $p_{1}, p_{2}, p_{3}$ satisfying

$$
\max _{1 \leq j \leq 3} p_{j} \leq 3|b|+\max \left\{3,\left|a_{1}\right|,\left|a_{2}\right|,\left|a_{3}\right|\right\}^{B} .
$$

Obviously, $B$ is the only relevant constant in (1.4). It is easy to see [LT2, p. 125] that $B$ must be larger than 1 . So, if we are not concerned about

1991 Mathematics Subject Classification: 11P32, 11P55, $11 \mathrm{D} 04$. 
the numerical value of $B$, Theorem 0 qualitatively settles Baker's problem on the bound for small prime solutions of the equation (1.1). Therefore, it remains to estimate the infimum $\mathcal{B}$ for all possible values of the constant $B$ in (1.4) which is now called the Baker constant. Plainly, the determination of $\mathcal{B}$ will completely settle the above-mentioned Baker problem.

Our investigation on the estimate for $\mathcal{B}$ is motivated not only by the Baker problem but also by the following interesting discoveries.

It was shown in [LT1, p. 596 and LT2, §2] that Theorem 0 contains the well-known Linnik Theorem [L] on the smallest prime in an arithmetic progression, namely, for any positive integers $l, q$ with $l \leq q$ and $(l, q)=$ 1 , the smallest prime $P(l, q)$ in the arithmetic progression $l+k q$ satisfies $P(l, q)<C q^{L}$ where $C$ and $L$ are some positive absolute constants. The infimum $\mathfrak{L}$ for all possible values of $L$ is called the Linnik constant. It was shown in $[\mathrm{LT} 2, \S 2]$ that $\mathcal{B} \geq \mathfrak{L}$. Many authors (see Table 1 in $[\mathrm{H}-\mathrm{B}]$ ) investigated the numerical bounds for $\mathfrak{L}$ while very little has been known for $\mathcal{B}$. The first numerical result for $\mathcal{B}$ was obtained by Choi [Cho]: $\mathcal{B} \leq 4190$. In the present paper we prove that $\mathcal{B} \leq 45$ in the following theorem.

ThEOREM 1. Assume conditions (1.2) and (1.3). If not all $a_{1}, a_{2}, a_{3}$ are of the same sign then there is an absolute constant $C>0$ such that the equation (1.1) has a prime solution $p_{1}, p_{2}, p_{3}$ satisfying

$$
\max _{1 \leq j \leq 3}\left|a_{j}\right| p_{j} \leq C \max \left\{|b|,\left(\max \left\{\left|a_{1}\right|,\left|a_{2}\right|,\left|a_{3}\right|\right\}\right)^{45}\right\} .
$$

That is, $\mathcal{B} \leq 45$.

Remark 1. Assuming the Generalized Riemann Hypothesis, it was shown in [CLT] that $\mathcal{B} \leq 4$.

Remark 2. Similar to Theorem 1 , we can prove that if all $a_{1}, a_{2}, a_{3}$ are positive and satisfy (1.2) and (1.3) then there is an absolute constant $C>0$ such that the equation (1.1) is solvable if $b \geq C\left(\max \left\{a_{1}, a_{2}, a_{3}\right\}\right)^{45}$. We prove this result simultaneously with our Theorem 1 in $\S 7$ and $\S 8$.

Our proof of the numerical result in Theorem 1 depends on an explicit zero-free region for Dirichlet $L$-functions and on an explicit zero-density estimate near the line $\sigma=1$ which will be given in $\S 2$ and $\S 3$ respectively. Basically, the results in $\S 2$ are due to Heath-Brown [H-B] but with some modifications in formulation for our use, and with a slight numerical improvement (see Lemma 2.1).

2. Zero-free regions for Dirichlet $L$-functions. The results obtained in this section which we shall use in our proof of Theorem 1 are in Proposition 2.3 (on the zero-free region), Lemma 2.5 (on two zeros) and Lemma 2.6 (on the Deuring-Heilbronn phenomenon). As usual, let $\chi(\bmod q)$ 
and $\chi^{0}(\bmod q)$ denote a Dirichlet character and the principal character modulo $q$ respectively. $L(s, \chi)$ denotes a Dirichlet $L$-function. $\varepsilon$ and $\varepsilon_{j}$ denote small positive numbers. Roughly speaking, this section is a reworking of $[\mathrm{H}-\mathrm{B}, \S \S 1-9]$. So we only give the details of the computational results but sketch the deductions. Instead of the function $\prod_{\chi(\bmod q)} L(s, \chi)$, which was considered in $[\mathrm{H}-\mathrm{B},(1.2)]$, we consider the zero-free regions of the function

$$
\Pi(s):=\prod_{q \leq Q} \prod_{\chi(\bmod q)}^{*} L(s, \chi)
$$

in the region $|\operatorname{Im} s| \leq C$ and $1 / 2 \leq \operatorname{Re} s \leq 1$, where $Q$ is a given sufficiently large positive number, $C$ is any positive constant, and the $*$ indicates that the product $\Pi^{*}$ is over all primitive characters $\chi(\bmod q)$. Similar to $[\mathrm{H}-\mathrm{B}$, $\S 6]$, we introduce the following notations. We put

$$
\mathcal{L}:=\log Q \text {. }
$$

Let $\varrho=\beta+i \gamma$ denote any zero of $\Pi(s)$ in the rectangle

$$
R:=\left\{s=\sigma+i t: 1-(3 \mathcal{L})^{-1} \log \log \mathcal{L} \leq \sigma \leq 1,|t| \leq C\right\} .
$$

Denote by $\varrho_{1}$ one of the above zeros for which $\beta$ is maximal, and let $\chi_{1}$ be a corresponding primitive character in $(2.1)$ such that $L\left(\varrho_{1}, \chi_{1}\right)=0$. Now, remove $L\left(s, \chi_{1}\right)$ and $L\left(s, \bar{\chi}_{1}\right)$ from $(2.1)$, and choose $\varrho_{2}$ to be one of the zeros of $\Pi(s)\left(L\left(s, \chi_{1}\right) L\left(s, \bar{\chi}_{1}\right)\right)^{-1}$ in $R$, for which $\beta$ is maximal. We take $\chi_{2}$ to be a primitive character in $(2.1)$ for which $L\left(\varrho_{2}, \chi_{2}\right)=0$. Then by arguments similar to those in [H-B, Lemma 6.1] we see that if a primitive character $\chi$ is different from $\chi_{1}, \bar{\chi}_{1}$, then every zero $\varrho$ of $L(s, \chi)$ satisfies

$$
\operatorname{Re} \varrho \leq \operatorname{Re} \varrho_{2} \quad \text { or } \quad|\operatorname{Im} \varrho| \geq 10 C \text {. }
$$

Moreover, $\chi_{1} \neq \chi_{2}, \bar{\chi}_{2}$. Next, we define the zero $\varrho^{\prime}$ of $L\left(s, \chi_{1}\right)$ in $R$ by one of the following three mutually exclusive conditions:

(i) If $\varrho_{1}$ is a repeated zero, then we choose $\varrho^{\prime}=\varrho_{1}$.

(ii) If $\varrho_{1}$ is simple and if $\chi_{1}$ is real and $\varrho_{1}$ is complex, then we choose $\varrho^{\prime} \neq \varrho_{1}, \bar{\varrho}_{1}$ in $R$ such that $\operatorname{Re} \varrho^{\prime}$ is maximal.

(iii) In the remaining cases, we choose $\varrho^{\prime} \neq \varrho_{1}$ in $R$ such that $\operatorname{Re} \varrho^{\prime}$ is maximal.

As in [H-B, (6.2)], we put

$$
\begin{aligned}
\varrho_{k} & :=\beta_{k}+i \gamma_{k}, \quad \beta_{k}:=1-\mathcal{L}^{-1} \lambda_{k}, \quad k=1,2, \\
\varrho^{\prime} & :=\beta^{\prime}+i \gamma^{\prime}, \quad \beta^{\prime}:=1-\mathcal{L}^{-1} \lambda^{\prime} .
\end{aligned}
$$

We first give a slight improvement on [H-B, Lemma 9.5] for the case $h=4$ there. Instead of [H-B, (9.15)], we start from the inequality

$$
0 \leq(1+\cos x)(1+2 \cos x)^{2}=5+8 \cos x+4 \cos 2 x+\cos 3 x .
$$


Let $f$ be the function defined as in [H-B, Condition 1, p. 280 and Condition 2, p. 286] and let $F$ be the Laplace transform of $f$, that is, for any complex $z$ put

$$
F(z):=\int_{0}^{\infty} e^{-z t} f(t) d t .
$$

Similar to [H-B, (9.16)], by (2.4) we get

$0 \leq 5 K\left(\beta_{1}, \chi_{0}\right)+8 K\left(\beta_{1}+i \gamma_{1}, \chi_{1}\right)+4 K\left(\beta_{1}+2 i \gamma_{1}, \chi_{1}^{2}\right)+K\left(\beta_{1}+3 i \gamma_{1}, \chi_{1}^{3}\right)$,

where $K(\beta+i \gamma, \chi)$ is defined as in [H-B, p. 285]. Since $h=4$, we have $\chi_{1}^{n} \neq \chi_{0}$ for $n=2,3$. Thus, by (2.3) and [H-B, Lemma 5.2] with $\phi=1 / 4$ defined as in [H-B, Lemma 2.5], we get

$$
\begin{aligned}
K\left(\beta_{1}+n i \gamma_{1}, \chi_{1}^{n}\right) & \leq f(0)(1 / 8+\varepsilon) \mathcal{L} \quad(n=2,3), \\
K\left(\beta_{1}+i \gamma_{1}, \chi_{1}\right) & \leq-F(0) \mathcal{L}+f(0)(1 / 8+\varepsilon) \mathcal{L} .
\end{aligned}
$$

Moreover, [H-B, Lemma 5.3] yields $K\left(\beta_{1}, \chi_{0}\right) \leq F\left(-\lambda_{1}\right) \mathcal{L}+\varepsilon f(0) \mathcal{L}$. Gathering together the above, we get

$$
5 F\left(-\lambda_{1}\right)-8 F(0)+(13 / 8) f(0)+\varepsilon \geq 0 .
$$

Now we use the function $f$ specified as in [H-B, Lemmas 7.1 and 7.5] with $k=8 / 5$. This yields $\theta=1.2161 \ldots$ and $\lambda_{1}^{-1} \cos ^{2} \theta \leq 13 / 40+\varepsilon$, whence $\lambda_{1} \geq 0.3711$. Replacing the 0.348 for the case $h=4$ in [H-B, Lemma 9.5] by 0.3711 , we see that the lower bound for $\lambda_{1}$ there now becomes 0.364 . Thus, by [H-B, Lemmas $8.4,8.8$ and 9.5], we can obtain a slight improvement on [H-B, Theorem 1] as in Lemma 2.1 below.

Lemma 2.1. For any constant $C>0$, there exists a $K(C)>0$ depending on $C$ only such that if $Q \geq K(C)$, then the function $\prod_{\chi(\bmod q)} L(s, \chi)$ with fixed $q \leq Q$ has at most one zero in the region $\sigma \geq 1-0.364 / \mathcal{L},|t| \leq C$. Such a zero, if it exists, is real and simple, and corresponds to a non-principal real character.

Lemma 2.2. Suppose that $\chi_{1}\left(\bmod q_{1}\right)$ and $\chi_{2}\left(\bmod q_{2}\right)$ are distinct, nonprincipal, primitive, real characters with $q_{1}, q_{2} \leq Q$, and that $\beta_{1}, \beta_{2}<1$ are real numbers satisfying $L\left(\beta_{1}, \chi_{1}\right)=L\left(\beta_{2}, \chi_{2}\right)=0$. Then $\min \left\{\beta_{1}, \beta_{2}\right\} \leq$ $1-0.4045 / \mathcal{L}$.

P r o of. Denote by $\chi_{\left[q_{1}, q_{2}\right]}^{0}$ the principal character modulo $\left[q_{1}, q_{2}\right]$. Then $L\left(\beta_{1}, \chi_{1} \chi_{\left[q_{1}, q_{2}\right]}^{0}\right)=L\left(\beta_{2}, \chi_{2} \chi_{\left[q_{1}, q_{2}\right]}^{0}\right)=0$. In view of $\chi_{1} \chi_{\left[q_{1}, q_{2}\right]}^{0} \neq \chi_{2} \chi_{\left[q_{1}, q_{2}\right]}^{0}$ and $\left[q_{1}, q_{2}\right] \leq Q^{2}$, we can deduce from [H-B, Table 6] that $\min \left\{\beta_{1}, \beta_{2}\right\} \leq$ $1-0.809 / \log Q^{2} \leq 1-0.809 /(2 \mathcal{L})$, as desired.

The combination of Lemmas 2.1 and 2.2 trivially implies 
Proposition 2.3. For any constant $C>0$, there exists a $K(C)>0$ depending on $C$ only such that if $Q \geq K(C)$, then the function $\Pi(s)$ defined by (2.1) has at most one zero in the region $\sigma \geq 1-0.364 / \mathcal{L},|t| \leq C$. Such a zero $\widetilde{\beta}$, if it exists, is real and simple, and corresponds to a non-principal, real, primitive character $\tilde{\chi}$ to a modulus $\widetilde{r} \leq Q . \widetilde{\beta}$ is called the Siegel zero or the exceptional zero.

The following is devoted to give a region in which $\Pi(s)$ has at most two zeros (see Lemma 2.5). We make use of the bounds for $\lambda^{\prime}$ in [H-B, Tables 2 to 4 and Table 8]. So we only need to give lower bounds for $\lambda_{2}$. Without loss of generality, we may assume that $\lambda_{2} \leq \lambda^{\prime}$, for otherwise the lower bound for $\lambda^{\prime}$ can serve as that for $\lambda_{2}$. As in [H-B, $\S 8$ and $\left.\S 9\right]$, we separate the arguments into two cases according as either both $\chi_{1}$ and $\varrho_{1}$ are real or not.

CASE I. $\chi_{1}$ and $\varrho_{1}$ are all real. We argue according to whether $\chi_{2}^{4}=\chi_{0}$ or $\chi_{2}^{4} \neq \chi_{0}$.

(i) $\chi_{2}^{4}=\chi_{0}$. We use the result (2.9) below, which is similar to [H-B, Lemma 8.5]. To prove (2.9), we use similar arguments to those of [H-B, Lemma 6.2]. Note that $\chi_{1} \chi_{2}$ and $\chi_{1} \bar{\chi}_{2}$ are non-principal characters to the modulus $\left[q_{1}, q_{2}\right] \leq Q^{2}$, and so [H-B, (6.5) and (6.6)] should be modified to (2.6) and (2.7) below respectively:

$$
\begin{aligned}
K\left(\beta_{1}+i \gamma_{1}+i \gamma_{2}, \chi_{1} \chi_{2}\right) & \leq f(0)\left((1 / 2) \phi\left(\chi_{1} \chi_{2}\right)+\varepsilon\right) \log Q^{2} \\
& \leq f(0)\left((1 / 2) 2 \phi\left(\chi_{1} \chi_{2}\right)+\varepsilon\right) \mathcal{L}
\end{aligned}
$$

and

$$
K\left(\beta_{1}+i \gamma_{1}-i \gamma_{2}, \chi_{1} \bar{\chi}_{2}\right) \leq f(0)\left((1 / 2) \cdot 2 \phi\left(\chi_{1} \bar{\chi}_{2}\right)+\varepsilon\right) \mathcal{L} .
$$

And consequently, by [H-B, (6.4) and (6.7) to (6.9)], we may modify the $\psi$ in $[\mathrm{H}-\mathrm{B},(6.10)]$ as

$$
\begin{aligned}
\psi & =(1 / 2) \phi\left(\chi_{1}\right)+(1 / 2) \phi\left(\chi_{2}\right)+(1 / 4)\left\{2 \phi\left(\chi_{1} \chi_{2}\right)\right\}+(1 / 4)\left\{2 \phi\left(\chi_{1} \bar{\chi}_{2}\right)\right\} \\
& \leq 1 / 2
\end{aligned}
$$

since $\chi_{1}$ and $\chi_{2}$ are of finite order and then by the definition of $\phi$ in $[\mathrm{H}-\mathrm{B}$, Lemma 2.5], all $\phi$ of the above are $1 / 4$. Thus, similar to [H-B, Lemma 8.5] we have

$$
F\left(-\lambda_{2}\right)-F\left(\lambda_{1}-\lambda_{2}\right)-F(0)+(1 / 2+\varepsilon) f(0) \geq 0 .
$$

We apply (2.9) with the function $f$ specified as in [H-B, Lemmas 7.1 and $7.5]$ with $k=2$, that is, $\theta=0.9873 \ldots$ In order to specify $f$ we must also select $\lambda$ there, and we make a variety of choices, depending on the size of $\lambda_{1}$. Let $\lambda_{1}$ satisfy $0 \leq \lambda_{1} \leq b$ and $\lambda=\lambda(b)$ be specified. Note that by (2.5) the function 


$$
F\left(-\lambda_{2}\right)-F\left(\lambda_{1}-\lambda_{2}\right)=\int_{0}^{\infty} f(t) e^{\lambda_{2} t}\left(1-e^{-\lambda_{1} t}\right) d t
$$

is increasing with respect to both $\lambda_{1}$ and $\lambda_{2}$. If we choose $\lambda_{2}(b)$ to give

$$
F\left(-\lambda_{2}(b)\right)-F\left(b-\lambda_{2}(b)\right)-F(0)+f(0) / 2=0,
$$

it then follows from (2.9) that $\lambda_{2} \geq \lambda_{2}(b)-\varepsilon$ whenever $0 \leq \lambda_{1} \leq b$ for $Q$ large enough. Table 1 below gives values for $b$ (as $\lambda_{1}$ ), for $\lambda(b)$ (as $\lambda$ ) and the calculated values a little below $\lambda_{2}(b)\left(\right.$ as $\left.\lambda_{2}\right)$.

Table 1. $\lambda_{2}$ for real $\chi_{1}$ and $\varrho_{1}, \chi_{2}^{4}=\chi_{0}$ (cf. Table 6 in [H-B])

\begin{tabular}{lcc|ccc|ccc}
\hline \multicolumn{1}{c}{$\lambda_{1}$} & $\lambda$ & $\lambda_{2}$ & $\lambda_{1}$ & $\lambda$ & $\lambda_{2}$ & $\lambda_{1}$ & $\lambda$ & $\lambda_{2}$ \\
\hline 0.003 & 0.83 & 5.61 & 0.128 & 0.693 & 1.83 & 0.30 & 0.62 & 1.02 \\
0.0035 & 0.83 & 5.46 & 0.16 & 0.676 & 1.61 & 0.35 & 0.60 & 0.89 \\
0.005 & 0.82 & 5.11 & 0.18 & 0.67 & 1.52 & 0.40 & 0.58 & 0.78 \\
0.008 & 0.81 & 4.62 & 0.20 & 0.66 & 1.42 & 0.45 & 0.57 & 0.68 \\
0.016 & 0.79 & 3.93 & 0.22 & 0.65 & 1.32 & 0.50 & 0.56 & 0.59 \\
0.032 & 0.766 & 3.22 & 0.25 & 0.64 & 1.20 & 0.53 & 0.54 & 0.55 \\
0.064 & 0.733 & 2.50 & 0.28 & 0.63 & 1.10 & 0.539 & 0.54 & 0.539 \\
\hline
\end{tabular}

(ii) $\chi_{2}^{4} \neq \chi_{0}$. Then none of the characters $\chi_{2}, \chi_{1} \chi_{2}, \chi_{2}^{2}$ or $\chi_{1} \chi_{2}^{2}$ is equal to $\chi_{0}$ or $\chi_{1}$. Noting that the modulus of $\chi_{1} \chi_{2}$ and $\chi_{1} \chi_{2}^{2}$ is $\left[q_{1}, q_{2}\right] \leq Q^{2}$, similar to the modification of $\psi$ in (2.8), we have for any constant $k \geq 0$ and any $\varepsilon>0$,

$$
\left(k^{2}+1 / 2\right)\left\{F\left(-\lambda_{2}\right)-F\left(\lambda_{1}-\lambda_{2}\right)\right\}-2 k F(0)+(\psi+\varepsilon) f(0) \geq 0,
$$

where the $\psi$ corresponding to that in $[\mathrm{H}-\mathrm{B},(8.10)]$ is modified to be $\psi=$ $\left(k^{2}+8 k+2.5\right) / 8$. Now we use $f$ in $[\mathrm{H}-\mathrm{B}$, Lemma 7.5] with $\theta=1$ and let $k=0.98-0.14 \lambda_{1}$. Then (2.10) yields the following Table 2 in a similar way as we get Table 1 from (2.9).

Table 2. $\lambda_{2}$ for real $\chi_{1}$ and $\varrho_{1}, \chi_{2}^{4} \neq \chi_{0}$ (cf. Table 7 in $[\mathrm{H}-\mathrm{B}]$ )

\begin{tabular}{lll|ccc}
\hline \multicolumn{1}{c}{$\lambda_{1}$} & \multicolumn{1}{c|}{$\lambda$} & \multicolumn{1}{c|}{$\lambda_{2}$} & $\lambda_{1}$ & $\lambda$ & $\lambda_{2}$ \\
\hline 0.0025 & 0.65 & 4.55 & 0.4 & 0.46 & 0.691 \\
0.066 & 0.566 & 2.00 & 0.45 & 0.45 & 0.615 \\
0.2 & 0.5 & 1.16 & 0.48 & 0.44 & 0.578 \\
0.306 & 0.477 & 0.867 & 0.5 & 0.43 & 0.557 \\
0.365 & 0.46 & 0.75 & 0.527 & 0.42 & 0.527 \\
\hline
\end{tabular}

CASE II. Either $\chi_{1}$ or $\varrho_{1}$ (or both) is complex. We separate the arguments into three cases:

(i) $\chi_{1}^{2} \neq \chi_{0}, \chi_{2}, \bar{\chi}_{2}$. Note that the modulus of $\chi_{1} \chi_{2}, \bar{\chi}_{1} \chi_{2}, \chi_{1}^{2} \chi_{2}$ and $\bar{\chi}_{1}^{2} \chi_{2}$ is $\left[q_{1}, q_{2}\right] \leq Q^{2}$. Then similar to [H-B, Lemma 9.2] we can apply the same 
arguments as in the above Case $\mathrm{I}(\mathrm{i})$ to the first inequality in $[\mathrm{H}-\mathrm{B}, \mathrm{p} .306]$ with $j=2$ to obtain

$$
\left(k^{2}+1 / 2\right)\left\{F\left(-\lambda_{1}\right)-F\left(\lambda_{2}-\lambda_{1}\right)\right\}-2 k F(0)+(\psi+\varepsilon) f(0) \geq 0
$$

where $\psi=\frac{1}{6}\left(k^{2}+6 k+2\right)$. Now we take $f$ in [H-B, Lemma 7.1] with $\theta=1$ and let $k=0.78+0.1 \lambda_{1}$. With this choice, we get

Table 3. $\lambda_{2}$ in the complex case, $\chi_{2} \neq \chi_{1}^{2}, \bar{\chi}_{1}^{2}$ and $\chi_{1}^{2} \neq \chi_{0}(\mathrm{cf}$. Table 9 in $[\mathrm{H}-\mathrm{B}])$

\begin{tabular}{lcl|lcc}
\hline \multicolumn{1}{c}{$\lambda_{1}$} & $\lambda_{2}$ & \multicolumn{1}{c|}{$\lambda$} & $\lambda_{1}$ & $\lambda_{2}$ & $\lambda$ \\
\hline 0.348 & 0.700 & 0.35 & 0.45 & 0.563 & 0.39 \\
0.36 & 0.681 & 0.36 & 0.48 & 0.531 & 0.39 \\
0.40 & 0.624 & 0.37 & 0.505 & 0.505 & 0.40 \\
\hline
\end{tabular}

(ii) $\chi_{2}^{2} \neq \chi_{0}, \chi_{1}, \bar{\chi}_{1}$. By reversing the roles of $\chi_{1}$ and $\chi_{2}$ in Case II(i), we get

$$
\begin{aligned}
& \left(k^{2}+1 / 2\right)\left\{F\left(-\lambda_{1}\right)-F(0)\right\} \\
& \quad-2 k F\left(\lambda_{2}-\lambda_{1}\right)+\left(k^{2}+6 k+2\right) f(0) / 6+\varepsilon \geq 0 .
\end{aligned}
$$

We take $k=0.94-0.1 \lambda_{1}$ and choose $f$ in [H-B, Lemma 7.1] with $\theta=1$. With this choice of $k$ and $\theta$, from (2.11) we get the following Table 4 parallel to [H-B, Table 10] by choosing the $\delta$ in [H-B, p. 307] to be 0.001 .

Table 4. $\lambda_{2}$ in the complex case, $\chi_{1} \neq \chi_{2}^{2}, \bar{\chi}_{2}^{2}$ and $\chi_{2}^{2} \neq \chi_{0}$ (cf. Table 10 in $\left.[\mathrm{H}-\mathrm{B}]\right)$

\begin{tabular}{lcc|ccc}
\hline$\lambda_{1}$ & $\lambda_{2}$ & $\lambda$ & $\lambda_{1}$ & $\lambda_{2}$ & $\lambda$ \\
\hline 0.348 & 0.587 & 0.38 & 0.45 & 0.530 & 0.39 \\
0.36 & 0.578 & 0.38 & 0.48 & 0.516 & 0.40 \\
0.40 & 0.555 & 0.38 & 0.504 & 0.504 & 0.40 \\
\hline
\end{tabular}

(iii) Both $\chi_{2}^{2}=\chi_{0}, \chi_{1}$ or $\bar{\chi}_{1}$ and $\chi_{1}^{2}=\chi_{0}, \chi_{2}$ or $\bar{\chi}_{2}$ hold. This happens only when $\chi_{1}$ and $\chi_{2}$ have order 5 or less. To cover this situation, we can use [H-B, Lemma 6.2] directly, with the $\psi$ in $[\mathrm{H}-\mathrm{B},(6.10)]$ being modified to be as (2.8). Hence we can produce the following

Table 5. $\lambda_{2}$ in the complex case (cf. Table 11 in $[\mathrm{H}-\mathrm{B}])$

\begin{tabular}{lcc|ccc}
\hline$\lambda_{1}$ & $\lambda_{2}$ & $\lambda$ & \multicolumn{1}{c}{$\lambda_{1}$} & $\lambda_{2}$ & $\lambda$ \\
\hline 0.34 & 0.712 & 0.49 & 0.48 & 0.583 & 0.53 \\
0.36 & 0.691 & 0.49 & 0.5 & 0.568 & 0.53 \\
0.4 & 0.652 & 0.51 & 0.539 & 0.539 & 0.54 \\
0.45 & 0.608 & 0.52 & & & \\
\hline
\end{tabular}


Comparison of Tables 1 to 5 shows that Table 4 gives the weakest result. Hence Table 4 applies in all cases. We summarize this as follows.

Lemma 2.4. The bounds given in Table 4 can be applied in all cases. In particular, $\lambda_{2} \geq 0.504$.

The combination of [H-B, Tables 4 and 8] and Lemma 2.4 together with the definition of $\varrho_{1}, \varrho_{2}$ and $\varrho^{\prime}$ implies

Lemma 2.5. For any constant $C>0$, there exists a $K(C)>0$ depending on $C$ only such that if $Q \geq K(C)$, then the function $\Pi(s)$ defined by (2.1) has at most two zeros in the region $\sigma \geq 1-0.504 / \mathcal{L},|t| \leq C$. Moreover, the bounds in Table 4 can be applied in all cases.

Lemma 2.6. If the exceptional zero $\widetilde{\beta}$ in Proposition 2.3 does indeed exist, then for any constant $c$ with $0<c<1$ and for any small $\varepsilon>0$ there is a $K(c, \varepsilon)>0$ depending on $c$ and $\varepsilon$ only such that for any zero $\varrho=\beta+i \gamma \neq \widetilde{\beta}$ (corresponding to $\chi(\bmod q)$ ) of the function $\Pi(s)$ defined by (2.1) we have

$$
\beta \leq 1-\min \left\{\frac{c}{6}, \frac{(1-c)(2 / 3-\varepsilon)}{\log ([\widetilde{r}, q]|\gamma|)} \log \left(\frac{(1-c)(2 / 3-\varepsilon)}{(1-\widetilde{\beta}) \log ([\widetilde{r}, q]|\gamma|)}\right)\right\}
$$

if $[\widetilde{r}, q]|\gamma|>K(c, \varepsilon)$. Moreover, for any positive $\varepsilon$ there exists a constant $c(\varepsilon)>0$ depending on $\varepsilon$ only such that

$$
1-0.364 / \mathcal{L} \leq \widetilde{\beta} \leq 1-c(\varepsilon) \widetilde{r}^{-\varepsilon} .
$$

Proof. (2.12) is a direct consequence of [G1, Theorem 10.1]. For the second inequality in (2.13), one can see, for example, [D, p. 127, (5)].

3. The zero-density estimates near the line $\sigma=1$. In this section, we give an explicit zero-density estimate for $L$-functions $L(s, \chi)$ near the line $\operatorname{Re} s=1$ with $|\operatorname{Im} s| \leq C$, where $C$ is any absolute constant. The result is

Lemma 3.1. For any absolute constant $C>0$, let $\alpha=1-\lambda / \mathcal{L}$ and let $N^{*}(\alpha, Q, C)$ be defined as in (3.1) below. Then for $Q \geq K(C)$ which is a positive constant depending on $C$ only, we have

$$
N^{*}(\alpha, Q, C) \leq N_{j}^{*} \quad(j=4,5,6,7,8)
$$

where 


$$
\begin{aligned}
& \frac{8.86706}{\lambda}\left(\exp (4.31403 \lambda)-\frac{\exp (3.15402 \lambda)-\exp (2.32002 \lambda)}{0.834 \lambda}\right):=N_{8}^{*} \\
& \text { if } 0.504<\lambda \leq 0.696 \text {, } \\
& \frac{26.93}{\lambda}\left(\exp (4.28374 \lambda)-\frac{\exp (3.19253 \lambda)-\exp (2.42653 \lambda)}{0.766 \lambda}\right):=N_{7}^{*} \\
& \text { if } 0.696<\lambda \leq 1 \text {, } \\
& \frac{50.36}{\lambda}\left(\exp (3.753506 \lambda)-\frac{\exp (2.747904 \lambda)-\exp (2.160104 \lambda)}{0.58 \lambda}\right):=N_{6}^{*} \\
& \text { if } 1<\lambda \leq 2, \\
& \frac{167.67}{\lambda}\left(\exp (3.116796 \lambda)-\frac{\exp (2.223794 \lambda)-\exp (1.869794 \lambda)}{0.354 \lambda}\right):=N_{5}^{*} \\
& \text { if } 2<\lambda \leq 6 \text {, } \\
& 42.54\left(1+\frac{35.385}{\lambda}\right)\left(\exp (2.87538 \lambda)-\frac{\exp (2.07176 \lambda)-\exp (1.92136 \lambda)}{0.1504 \lambda}\right) \\
& :=N_{4}^{*} \quad \text { if } 6<\lambda \leq \log \log \mathcal{L} \text {. }
\end{aligned}
$$

To prove Lemma 3.1, we first give some notations. For $1 \leq j \leq 4$, let $h_{j}$ be absolute constants satisfying $1<h_{1}<h_{2}<h_{3}$, and their exact values will be specified later in each individual case, e.g. in (3.17), (3.26). Put

$$
\left\{\begin{array}{l}
z_{j}:=Q^{h_{j}} \quad \text { for } 1 \leq j \leq 4, \\
\alpha:=1-\lambda / \mathcal{L} \quad \text { for } 0.364 \leq \lambda \leq \log \log \mathcal{L}, \\
D:=\{s=\sigma+i t: \alpha \leq \sigma<1-0.364 / \mathcal{L},|t| \leq C\} \\
N(\chi, \alpha, C):=\text { number of zeros of } L(s, \chi) \text { in } D, \\
N^{*}(\alpha, Q, C):=\sum_{q \leq Q} \sum_{\chi(\bmod q)}^{*} N(\chi, \alpha, C),
\end{array}\right.
$$

where $\sum_{\chi}^{*}(\bmod q)$ denotes the summation over all primitive characters $\chi(\bmod q)$; and we use the symbols $\theta_{d}(q)$ and $G(q)$ defined as in [LLW, (3.2)].

We now present two preliminary lemmas.

Lemma 3.2. For any $C>0$ let $Q \geq K(C)$ which is a positive constant depending on $C$ only. Suppose $\chi_{1}\left(\bmod q_{1}\right)$ and $\chi_{2}\left(\bmod q_{2}\right)$ are two primitive characters with $q_{1}, q_{2} \leq Q$. Let $s=\sigma+i t$ with $|t| \leq C$ and $0<\sigma \leq 3(\log \log \mathcal{L}) / \mathcal{L}$. Define $E_{0}=1$ if $\chi_{1}=\chi_{2}$ and $E_{0}=0$ if $\chi_{1} \neq \chi_{2}$. Then, if $3 / 4+2 h_{4}+\varepsilon<h_{1}<h_{2}$ we have 


$$
\begin{aligned}
\sum_{z_{1}<n \leq z_{3}}\left(\sum_{d \mid n} \theta_{d}\left(q_{1}\right)\right)\left(\sum_{d \mid n} \theta_{d}\left(q_{2}\right)\right) \chi_{1}(n) \bar{\chi}_{2}(n) n^{-s-1} & \\
= & \frac{E_{0} \varphi\left(\left[q_{1}, q_{2}\right]\right)}{G\left(\left[q_{1}, q_{2}\right]\right)\left[q_{1}, q_{2}\right]} \int_{\log z_{1}}^{\log z_{3}} e^{-s x} d x+O\left(\mathcal{L}^{-1}\right) .
\end{aligned}
$$

Proof. As [LLW, Lemma 11], the lemma can be proved by the same arguments as in the proof of [Che, Lemma 8]. The replacement of the $3 / 8$ in [LLW, Lemma 11] by the present $3 / 4$ is due to the fact that the $z_{j}=\left(P^{2} T\right)^{h_{j}}$ in [LLW, (3.1)] is now replaced by the $z_{j}=Q^{h_{j}}$ defined as in (3.1).

LEMMA 3.3. Let $\chi$ be a non-principal character modulo $q \leq Q$, and let $n_{1}, \ldots, n_{5}$ be the number of zeros of $L(s, \chi)$ in the intersections of $D$ (in (3.1)) with the following regions $R_{1}, \ldots, R_{5}$ respectively:

$$
R_{j}: \quad 1-\lambda / \mathcal{L} \leq \sigma \leq 1-0.364 / \mathcal{L},\left|t-t_{j}\right| \leq \tau_{j} / \mathcal{L},
$$

where $t_{1}, \ldots, t_{5}$ are any real numbers and $\tau_{1}, \ldots, \tau_{5}$ are $20,13.6,9.1,6.64$, 1.06 respectively. Then

$n_{1} \leq(0.2167)(\lambda+35.385)$ for $6<\lambda \leq \log \log \mathcal{L}, \quad n_{2} \leq 6$ for $2<\lambda \leq 6$,

$n_{3} \leq 4$ for $1<\lambda \leq 2, \quad n_{4} \leq 3$ for $0.696<\lambda \leq 1$,

$n_{5} \leq 1$ for $0.504<\lambda \leq 0.696$.

Proof. Note that for any real $\sigma$ and $t$ with $\sigma>1$,

$$
-\operatorname{Re}\left(\zeta^{\prime} / \zeta\right)(\sigma)-\operatorname{Re}\left(L^{\prime} / L\right)(\sigma+i t, \chi) \geq 0 .
$$

Thus from $-\left(\zeta^{\prime} / \zeta\right)(\sigma) \leq(\sigma-1)^{-1}+O(1)$ we get for $\sigma>1$,

$$
0 \leq(\sigma-1)^{-1}-\operatorname{Re}\left(L^{\prime} / L\right)(\sigma+i t, \chi)+O(1) .
$$

Taking $\sigma=1+20 / \mathcal{L}$ and $t=t_{1}$, by [H-B, Lemma 3.1 with $\left.\phi=1 / 3\right]$ and the definition of $R_{1}$, we get

$$
\frac{1}{20}+\frac{1}{6}+\varepsilon-n_{1} \min _{0.364 \leq \beta \leq \lambda}\left\{\frac{20+\beta}{(20+\beta)^{2}+400}\right\} \geq 0,
$$

where $\beta=(1-\operatorname{Re} \varrho) \mathcal{L}$ and $\varrho$ is a zero of $L(s, \chi)$ in $D \cap R_{1}$. Hence for $6<\lambda \leq \log \log \mathcal{L}$,

$$
\begin{aligned}
n_{1} & \leq\left(\frac{1}{20}+\frac{1}{6}+\varepsilon\right) \max _{0.364 \leq \beta \leq \lambda}\left\{\frac{(20+\beta)^{2}+400}{20+\beta}\right\} \\
& \leq\left(\frac{1}{20}+\frac{1}{6}+\varepsilon\right) \max \left\{\frac{20+\lambda^{2}+400}{20+\lambda}, \frac{(20.364)^{2}+400}{20.364}\right\} \\
& \leq(0.2167)(\lambda+35.385) .
\end{aligned}
$$

Similarly, taking the $\sigma$ and $t$ in $(3.2)$ as $\sigma=1+14.84 / \mathcal{L}, 1+11.8 / \mathcal{L}, 1+$ $9.49 / \mathcal{L}, 1+2.88 / \mathcal{L}$, and $t=t_{2}, \ldots, t_{5}$ respectively, we get by $(3.2),[\mathrm{H}-\mathrm{B}$, 
Lemma 3.1 with $\phi=1 / 3$ ] and the definition of $R_{2}, \ldots, R_{5}$,

$$
\begin{aligned}
n_{2} & \leq\left[\left(\frac{1}{14.84}+\frac{1}{6}+\varepsilon\right)\left(14.84+\lambda+\frac{(13.6)^{2}}{14.84+\lambda}\right)\right] \\
& \leq[6.955]=6 \text { for } 2<\lambda \leq 6 ; \\
n_{3} & \leq 4 \text { for } 1<\lambda \leq 2 ; \quad n_{4} \leq 3 \text { for } 0.696<\lambda \leq 1 ; \\
n_{5} & \leq 1 \text { for } 0.504<\lambda \leq 0.696,
\end{aligned}
$$

where $[x]$ denotes the greatest integer not exceeding $x$. The proof of Lemma 3.3 is complete.

We are now going to prove Lemma 3.1. Define for any complex $s$,

$$
\begin{aligned}
\kappa(s)= & s^{-2}\left(\left(\exp \left(-\left(1-\delta_{1}\right)\left(\log z_{1}\right) s\right)-\exp \left(-\left(\log z_{1}\right) s\right)\right) \delta_{3}\left(\log z_{3}\right)\right. \\
& \left.-\left(\exp \left(-\left(\log z_{3}\right) s\right)-\exp \left(-\left(1+\delta_{3}\right)\left(\log z_{3}\right) s\right)\right) \delta_{1}\left(\log z_{1}\right)\right),
\end{aligned}
$$

where $\delta_{1}, \delta_{3}$ are positive numbers with $0<\delta_{1}, \delta_{3}<1$. For a zero $\varrho_{0} \in D$, put

$$
M\left(\varrho_{0}\right):=\sum_{\varrho(\chi)}\left|\kappa\left(\varrho(\chi)+\bar{\varrho}_{0}-2 \alpha\right)\right|,
$$

where $\varrho(\chi)$ is any zero of $L(s, \chi)$ in $D$. Then, similar to the arguments leading to [LLW, (3.17)], it can be derived by the use of Lemma 3.2 and [LLW, Lemma 10, and Che, Lemma 4] that

$$
N^{*}(\alpha, Q, C) \leq \frac{1+\varepsilon}{2 \lambda\left(h_{2}-h_{1}\right)}\left(e^{2 h_{3} \lambda}-\frac{e^{2 h_{2} \lambda}-e^{2 h_{1} \lambda}}{2 \lambda\left(h_{2}-h_{1}\right)}\right) \frac{\max _{\varrho_{0}} M\left(\varrho_{0}\right)}{\delta_{1} \delta_{3} h_{1} h_{3} h_{4} \mathcal{L}^{3}},
$$

if one assumes that

$$
h_{1}<h_{2}, \quad h_{2}+h_{4}+3 / 8+\varepsilon<h_{3} \quad \text { and } \quad 2 h_{4}+3 / 4+\varepsilon<\left(1-\delta_{1}\right) h_{1} .
$$

In view of the definition of $D$ in (3.1), we have $\operatorname{Re}\left(\varrho_{1}+\bar{\varrho}_{2}\right)-2 \alpha \geq 0$ for any $\varrho_{1}, \varrho_{2} \in D$. Thus by $(3.3)$,

$$
\begin{aligned}
\left|\kappa\left(\varrho_{1}+\bar{\varrho}_{2}-2 \alpha\right)\right| & =\left|\int_{\log z_{3}}^{\left(1+\delta_{3}\right)} \int_{\left(1-\delta_{1}\right) \log z_{3}}^{\log z_{1}} \int_{\xi}^{\eta} e^{-\left(\varrho_{1}+\bar{\varrho}_{2}-2 \alpha\right) x} d x d \xi d \eta\right| \\
& \leq 2^{-1} \mathcal{L}^{3}\left\{\delta_{1} h_{1}\left(2 \delta_{3}+\delta_{3}^{2}\right) h_{3}^{2}-\delta_{3} h_{3}\left(2 \delta_{1}-\delta_{1}^{2}\right) h_{1}^{2}\right\} .
\end{aligned}
$$

For ease of notation, in due course of this section we write for any $\varrho(\chi)$, $\varrho_{0} \in D$,

$$
\varrho(\chi):=1-\beta_{\chi} \mathcal{L}^{-1}+i \gamma_{\chi} \mathcal{L}^{-1}, \quad \varrho_{0}:=1-\beta_{0} \mathcal{L}^{-1}+i \gamma_{0} \mathcal{L}^{-1} .
$$

We separate the arguments into the following five cases (i) to (v) according to the upper bounds for $\lambda$ at $1,2,6, \log \log \mathcal{L}$ and 0.696 respectively. 
(i) If $0.696<\lambda \leq 1$, then by taking $t_{4}=\gamma_{0} / \mathcal{L}$ in Lemma 3.3 we see that there are at most 3 zeros in $D \cap R_{4}$ (containing $\varrho_{0}$ ) and that

$$
\left|\gamma_{\chi}-\gamma_{0}\right| \geq 6.64
$$

for any $\varrho(\chi) \notin R_{4}$. On the other hand, we have trivially by the definition of $\kappa(s)$ in $(3.3),|\kappa(s)| \leq 2\left(\delta_{1} h_{1}+\delta_{3} h_{3}\right) \mathcal{L}|s|^{-2}$. Thus

$$
\begin{aligned}
& \sum_{\varrho(\chi) \in D-R_{4}}\left|\kappa\left(\varrho(\chi)+\bar{\varrho}_{0}-2 \alpha\right)\right| \\
& \leq 2\left(\delta_{1} h_{1}+\delta_{3} h_{3}\right) \mathcal{L}^{3} \sum_{\varrho(\chi) \in D-R_{4}}\left|\gamma_{\chi}-\gamma_{0}\right|^{-2} .
\end{aligned}
$$

Moreover, for any $a \neq-\beta_{\chi}$,

$$
\left(\gamma_{\chi}-\gamma_{0}\right)^{-2}=\left(\frac{a+\beta_{\chi}}{\left(\gamma_{\chi}-\gamma_{0}\right)^{2}}+\frac{1}{a+\beta_{\chi}}\right) \frac{a+\beta_{\chi}}{\left(a+\beta_{\chi}\right)^{2}+\left(\gamma_{\chi}-\gamma_{0}\right)^{2}} .
$$

Set $f(x, y)=x y^{-2}+x^{-1}$. For fixed $y, f(x, y)$ is increasing for $x \geq y$ and decreasing for $x<y$. Assume $a \geq 6.64$. Thus by (3.8) we obtain

$$
\begin{aligned}
\max _{0.364 \leq \beta_{\chi} \leq \lambda}\left\{\frac{a+\beta_{\chi}}{\left(\gamma_{\chi}-\gamma_{0}\right)^{2}}+\frac{1}{a+\beta_{\chi}}\right\} & \text { if } 6.64 \leq y \leq a+0.364, \\
\leq & \begin{cases}\frac{a+\lambda}{y^{2}}+\frac{1}{a+\lambda} & \max \left\{\frac{a+\lambda}{y^{2}}+\frac{1}{a+\lambda}, \frac{a+0.364}{y^{2}}+\frac{1}{a+0.364}\right\} \\
\frac{a+0.364}{y^{2}}+\frac{1}{a+0.364} & \text { if } a>0.364 \leq y \leq a+\lambda,\end{cases} \\
\leq & \text { if } 6.64 \leq y \leq((a+0.364)(a+\lambda))^{1 / 2}, \\
\frac{a+\lambda}{y^{2}+\frac{1}{a+\lambda}} & \text { if } y>((a+0.364)(a+\lambda))^{1 / 2} .
\end{aligned}
$$

Hence the last summation in (3.9) is

$$
\begin{aligned}
& \leq \max \left\{\max _{6.64 \leq y \leq((a+0.364)(a+\lambda))^{1 / 2}}\left\{\frac{a+\lambda}{y^{2}}+\frac{1}{a+\lambda}\right\},\right. \\
& \left.\quad \times \sum_{\varrho(\chi) \in D-R_{4}}^{\max _{y \geq(a+0.364)(a+\lambda))^{1 / 2}}}\left\{\frac{a+0.364}{y^{2}}+\frac{1}{a+0.364}\right\}\right\} \\
& \quad \frac{a+\beta_{\chi}}{\left(a+\beta_{\chi}\right)^{2}+\left(\gamma_{\chi}-\gamma_{0}\right)^{2}} .
\end{aligned}
$$

By (3.2) with $\sigma=1+a \mathcal{L}^{-1}, t=\gamma_{0} \mathcal{L}^{-1}$, and [H-B, Lemma 3.1 with $\phi=1 / 3$ ], 
the last summation in (3.10) can be estimated as, for $a+\lambda \geq 6.64$,

$$
\leq \frac{1}{a}-\frac{1}{a+\lambda}-\frac{E_{1}(a+\lambda)}{(a+\lambda)^{2}+(6.64)^{2}}+\frac{1}{6}+\varepsilon,
$$

where

\begin{tabular}{c|c|c|c}
\hline number of zeros of $L(s, \chi)$ in $D \cap R_{4}$ & one & two & three \\
\hline$E_{1}$ & 0 & 1 & 2 \\
\hline
\end{tabular}

Taking $a=7.136$ (so $a>6.64$ ), by (3.11) and $\lambda \leq 1$, (3.10) can be estimated as

$$
\begin{aligned}
\leq & \left(\frac{1}{a}-\frac{1}{a+\lambda}-\frac{E_{1}(a+\lambda)}{(a+\lambda)^{2}+(6.64)^{2}}+\frac{1}{6}+\varepsilon\right) \\
& \times \max \left\{\frac{a+\lambda}{(6.64)^{2}}+\frac{1}{a+\lambda}, \frac{1}{a+\lambda}+\frac{1}{a+0.364}\right\} \\
\leq & \left(\frac{1}{a}-\frac{1}{a+1}-\frac{E_{1}(a+1)}{(a+1)^{2}+(6.64)^{2}}+\frac{1}{6}+\varepsilon\right)\left(\frac{a+1}{(6.64)^{2}}+\frac{1}{a+1}\right) \\
\leq & f_{1}\left(E_{1}\right)
\end{aligned}
$$

where

\begin{tabular}{c|c|c|c}
\hline$E_{1}$ & 0 & 1 & 2 \\
\hline$f_{1}\left(E_{1}\right)$ & 0.05654 & 0.03386 & 0.011174 \\
\hline
\end{tabular}

Now by (3.4), (3.7), (3.9) and (3.12) we can summarize that, for $0.696<$ $\lambda \leq 1$,

$$
\begin{aligned}
\max _{\varrho_{0}} M\left(\varrho_{0}\right) \leq & \max _{0 \leq E_{1} \leq 2}\left\{( ( 1 + E _ { 1 } ) / 2 ) \left(\delta_{1} h_{1}\left(2 \delta_{3}+\delta_{3}^{2}\right) h_{3}^{2}\right.\right. \\
& \left.\left.-\delta_{3} h_{3}\left(2 \delta_{1}-\delta_{1}^{2}\right) h_{1}^{2}\right)+2\left(\delta_{1} h_{1}+\delta_{3} h_{3}\right) f_{1}\left(E_{1}\right)\right\} \mathcal{L}^{3} .
\end{aligned}
$$

Choose $\delta_{1}$ and $\delta_{3}$ satisfying the condition

$$
\delta_{1} h_{1}=\delta_{3} h_{3}=\left(4 f_{1}\left(E_{1}\right)\left(1+E_{1}\right)^{-1}\right)^{1 / 2} .
$$

By (3.5), (3.13) and (3.14) we get, for $0.696<\lambda \leq 1$,

$$
\begin{aligned}
& N^{*}(\alpha, Q, C) \\
\leq & \max _{0 \leq E_{1} \leq 2}(1+\varepsilon)\left(\left(1+E_{1}\right) / 2\right) \\
& \times \frac{\left\{\delta_{1} h_{1}\left(2 \delta_{3}+\delta_{3}^{2}\right) h_{3}^{2}-\delta_{3} h_{3}\left(2 \delta_{1}-\delta_{1}^{2}\right) h_{1}^{2}\right\}+2 f_{1}\left(E_{1}\right)\left(\delta_{1} h_{1}+\delta_{3} h_{3}\right)}{2 \lambda\left(h_{2}-h_{1}\right) \delta_{1} h_{1} \delta_{3} h_{3} h_{4}}
\end{aligned}
$$




$$
\begin{aligned}
& \times\left(e^{2 h_{3} \lambda}-\frac{e^{2 h_{2} \lambda}-e^{2 h_{1} \lambda}}{2 \lambda\left(h_{2}-h_{1}\right)}\right) \\
\leq & (1+\varepsilon) \max _{0 \leq E_{1} \leq 2} \frac{\left(1+E_{1}\right)\left(h_{3}-h_{1}\right)+4\left(\left(1+E_{1}\right) f_{1}\left(E_{1}\right)\right)^{1 / 2}}{2 \lambda\left(h_{2}-h_{1}\right) h_{4}} \\
& \times\left(e^{2 h_{3} \lambda}-\frac{e^{2 h_{2} \lambda}-e^{2 h_{1} \lambda}}{2 \lambda\left(h_{2}-h_{1}\right)}\right),
\end{aligned}
$$

providing (3.6) with $\delta_{1} h_{1}$ given as in (3.14). Let $h_{2}-h_{1}=x, h_{4}=y$. Then the optimal choices of $h$ 's are approximately

$$
\begin{aligned}
& h_{1}=\frac{3}{4}+\left(\frac{4 f_{1}\left(E_{1}\right)}{1+E_{1}}\right)^{1 / 2}+2 y+\varepsilon, \\
& h_{2}=h_{1}+x=\frac{3}{4}+\left(\frac{4 f_{1}\left(E_{1}\right)}{1+E_{1}}\right)^{1 / 2}+x+2 y+\varepsilon, \\
& h_{3}=\frac{3}{8}+x+y+h_{1}+\varepsilon=\frac{3}{8}+\frac{3}{4}+\left(\frac{4 f_{1}\left(E_{1}\right)}{1+E_{1}}\right)^{1 / 2}+x+3 y+2 \varepsilon, \\
& h_{4}=y .
\end{aligned}
$$

With these choices of $h$ 's, the last maximum in (3.15) corresponds to $E_{1}=2$. Hence in view of the definition of $f_{1}\left(E_{1}\right)$ in $(3.12),(3.15)$ is

$$
\leq(1+\varepsilon) \frac{3\left(h_{3}-h_{1}\right)+0.732361}{2 \lambda\left(h_{2}-h_{1}\right) h_{4}}\left(e^{2 h_{3} \lambda}-\frac{e^{2 h_{2} \lambda}-e^{2 h_{1} \lambda}}{2 \lambda\left(h_{2}-h_{1}\right)}\right),
$$

with

$$
\left\{\begin{array}{l}
h_{1}=3 / 4+(4(0.011174) / 3)^{1 / 2}+2 y+\varepsilon \\
h_{2}=3 / 4+(4(0.011174) / 3)^{1 / 2}+x+2 y+\varepsilon \\
h_{3}=3 / 8+3 / 4+(4(0.011174) / 3)^{1 / 2}+x+3 y+2 \varepsilon \\
h_{4}=y
\end{array}\right.
$$

Substituting (3.17) into (3.16), numerical experiments show that the optimal choices of $x$ and $y$ are approximately $x=0.383$ and $y=0.1706$. Substituting the above choices of $x$ and $y$ into (3.17) and then into (3.16) we conclude that for $0.696<\lambda \leq 1$,

$$
N^{*}(\alpha, Q, C) \leq \frac{26.93}{\lambda}\left(\exp (4.28374 \lambda)-\frac{\exp (3.19253 \lambda)-\exp (2.42653 \lambda)}{0.766 \lambda}\right)
$$

This is the second inequality for $N^{*}(\alpha, Q, C)$ in Lemma 3.1.

(ii) If $1<\lambda \leq 2$, then by taking $t_{3}=\gamma_{0} \mathcal{L}^{-1}$ in Lemma 3.3 we see that there are at most 4 zeros in $D \cap R_{3}$, and that $\left|\gamma_{\chi}-\gamma_{0}\right|>9.1$ for any $\varrho(\chi) \notin R_{3}$. Thus, completely similar to the arguments from (3.9) to (3.12) 
in the above case (i), we can obtain

$$
\begin{aligned}
& \sum_{\varrho(\chi) \in D-R_{3}}\left|\kappa\left(\varrho(\chi)+\varrho_{0}-2 \alpha\right)\right| \\
& \leq 2\left(\delta_{1} h_{1}+\delta_{3} h_{3}\right) \mathcal{L}^{3} \\
& \quad \times\left(\frac{1}{a}-\frac{1}{a+2}-\frac{E_{2}(a+2)}{(a+2)^{2}+(9.1)^{2}}+\frac{1}{6}+\varepsilon\right)\left(\frac{a+2}{(9.1)^{2}}+\frac{1}{a+2}\right) \\
& \leq 2\left(\delta_{1} h_{1}+\delta_{3} h_{3}\right) \mathcal{L}^{3} f_{2}\left(E_{2}\right),
\end{aligned}
$$

where

\begin{tabular}{c|c|c|c|c}
\hline$E_{2}$ & 0 & 1 & 2 & 3 \\
\hline$f_{2}\left(E_{2}\right)$ & 0.041771 & 0.29695 & 0.017619 & 0.005543 \\
\hline
\end{tabular}

providing $a=9.41$. Now choosing $\delta_{1}$ and $\delta_{3}$ by $\delta_{1} h_{1}=\delta_{3} h_{3}=\left(4 f_{2}\left(E_{2}\right) /\right.$ $\left.\left(1+E_{2}\right)\right)^{1 / 2}$, we can deduce, similar to (3.15) and (3.16),

$$
\begin{aligned}
N^{*}(\alpha, Q, C) & \\
\leq & (1+\varepsilon) \max _{0 \leq E_{2} \leq 3} \frac{\left(1+E_{2}\right)\left(h_{3}-h_{1}\right)+4\left(\left(1+E_{2}\right) f_{2}\left(E_{2}\right)\right)^{1 / 2}}{2 \lambda\left(h_{2}-h_{1}\right) h_{4}} \\
& \times\left(e^{2 h_{3} \lambda}-\frac{e^{2 h_{2} \lambda}-e^{2 h_{1} \lambda}}{2 \lambda\left(h_{2}-h_{1}\right)}\right) \\
\leq & (1+\varepsilon) \frac{4\left(h_{3}-h_{1}\right)+0.595611}{2 \lambda\left(h_{2}-h_{1}\right) h_{4}}\left(e^{2 h_{3} \lambda}-\frac{e^{2 h_{2} \lambda}-e^{2 h_{1} \lambda}}{2 \lambda\left(h_{2}-h_{1}\right)}\right),
\end{aligned}
$$

with the following approximately optimal choices of $h$ 's:

$$
\left\{\begin{array} { l } 
{ h _ { 1 } = 3 / 4 + ( 0 . 0 0 5 5 4 3 ) ^ { 1 / 2 } + 2 y + \varepsilon , \quad h _ { 2 } = h _ { 1 } + x , } \\
{ h _ { 3 } = h _ { 1 } + x + y + 3 / 8 + \varepsilon , \quad h _ { 4 } = y , }
\end{array} \quad \text { and } \quad \left\{\begin{array}{l}
x=0.2939 \\
y=0.1278
\end{array}\right.\right.
$$

With these choices of $h_{1}, \ldots, h_{4}$, from (3.18) we derive the third inequality for $N^{*}(\alpha, Q, C)$ in Lemma 3.1.

(iii) If $2<\lambda \leq 6$, then by taking $t_{2}=\gamma_{0} \mathcal{L}^{-1}$ in Lemma 3.3 we see that there are at most 6 zeros in $D \cap R_{2}$, and that $\left|\gamma_{\chi}-\gamma_{0}\right|>13.6$ for any $\varrho(\chi) \notin R_{2}$. Hence similar to case (i) we have

$$
\begin{aligned}
& \quad \sum_{\varrho(\chi) \in D-R_{2}}\left|\kappa\left(\varrho(\chi)+\bar{\varrho}_{0}-2 \alpha\right)\right| \\
& \leq 2\left(\delta_{1} h_{1}+\delta_{3} h_{3}\right) \mathcal{L}^{3} \\
& \quad \times\left(\frac{1}{a}-\frac{1}{a+6}-\frac{E_{3}(a+6)}{(a+6)^{2}+(13.6)^{2}}+\frac{1}{6}+\varepsilon\right)\left(\frac{a+6}{(13.6)^{2}}+\frac{1}{a+6}\right) \\
& \leq 2\left(\delta_{1} h_{1}+\delta_{3} h_{3}\right) \mathcal{L}^{3} f_{3}\left(E_{3}\right),
\end{aligned}
$$


where

\begin{tabular}{c|c|c|c|c|c|c}
\hline$E_{3}$ & 0 & 1 & 2 & 3 & 4 & 5 \\
\hline$f_{3}\left(E_{3}\right)$ & 0.029666 & 0.02426 & 0.018853 & 0.013447 & 0.00804 & 0.002633 \\
\hline
\end{tabular}

providing $a=12.8938$. Now choose $\delta_{1}$ and $\delta_{3}$ by $\delta_{1} h_{1}=\delta_{3} h_{3}=\left(4 f_{3}\left(E_{3}\right) /\right.$ $\left.\left(1+E_{3}\right)\right)^{1 / 2}$. Similar to (3.15) and (3.16), we can deduce for $2<\lambda \leq 6$,

$$
\begin{aligned}
& N^{*}(\alpha, Q, C) \\
& \leq(1+\varepsilon) \max _{0 \leq E_{3} \leq 5} \frac{\left(1+E_{3}\right)\left(h_{3}-h_{1}\right)+4\left(\left(1+E_{3}\right) f_{3}\left(E_{3}\right)\right)^{1 / 2}}{2 \lambda\left(h_{2}-h_{1}\right) h_{4}} \\
& \quad \times\left(e^{2 h_{3} \lambda}-\frac{e^{2 h_{2} \lambda}-e^{2 h_{1} \lambda}}{2 \lambda\left(h_{2}-h_{1}\right)}\right) \\
& \leq(1+\varepsilon) \frac{6\left(h_{3}-h_{1}\right)+0.502761}{2 \lambda\left(h_{2}-h_{1}\right) h_{4}}\left(e^{2 h_{3} \lambda}-\frac{e^{2 h_{2} \lambda}-e^{2 h_{1} \lambda}}{2 \lambda\left(h_{2}-h_{1}\right)}\right),
\end{aligned}
$$

with the following approximately optimal choices of $h$ 's:

$$
\left\{\begin{array}{l}
h_{1}=3 / 4+4(0.002633) / 6^{1 / 2}+2 y+\varepsilon, \quad h_{2}=h_{1}+x \\
h_{3}=h_{1}+x+y+3 / 8+\varepsilon, \quad h_{4}=y
\end{array}\right.
$$

and

$$
\left\{\begin{array}{l}
x=0.177 \\
y=0.0715
\end{array}\right.
$$

Therefore from (3.19) we derive the next-to-last inequality for $N^{*}(\alpha, Q, C)$ in Lemma 3.1.

(iv) If $6<\lambda \leq \log \log \mathcal{L}$, then similar to [LLW, $\S 3$, case (i)], by Lemma 3.3 we get

$$
\begin{aligned}
\max _{\varrho_{0}} M\left(\varrho_{0}\right) \leq & (0.2167)(\lambda+35.385)\left\{(1 / 2) \delta_{1} h_{1} \delta_{3} h_{3}\left(2 h_{3}-2 h_{1}+\delta_{1} h_{1}+\delta_{3} h_{3}\right)\right. \\
& \left.+(1 / 2)(\pi / 20)^{2}\left(\delta_{1} h_{1}+\delta_{3} h_{3}\right)\right\} \mathcal{L}^{3} .
\end{aligned}
$$

Then by (3.5),

$$
\begin{aligned}
& N^{*}(\alpha, Q, C) \\
& \leq(1+\varepsilon)(0.2167)(\lambda+35.385) \\
& \quad \times \frac{\left(2 h_{3}-2 h_{1}+\delta_{1} h_{1}+\delta_{3} h_{3}+(\pi / 20)^{2}\left(1 /\left(\delta_{1} h_{1}\right)+1 /\left(\delta_{3} h_{3}\right)\right)\right)}{4 \lambda\left(h_{2}-h_{1}\right) h_{4}} \\
& \quad \times\left(e^{2 h_{3} \lambda}-\frac{e^{2 h_{2} \lambda}-e^{2 h_{1} \lambda}}{2 \lambda\left(h_{2}-h_{1}\right)}\right)
\end{aligned}
$$




$$
\begin{aligned}
\leq & \frac{(1+\varepsilon)(0.2167)(\lambda+35.385)\left(2 h_{3}-2 h_{1}+\pi / 5\right)}{4 \lambda\left(h_{2}-h_{1}\right) h_{4}} \\
& \times\left(e^{2 h_{3} \lambda}-\frac{e^{2 h_{2} \lambda}-e^{2 h_{1} \lambda}}{2 \lambda\left(h_{2}-h_{1}\right)}\right)
\end{aligned}
$$

providing (3.6) with $\delta_{1} h_{1}=\delta_{3} h_{3}=\pi / 20$. Let $h_{2}-h_{1}=x, h_{4}=y$. Then the optimal choices of $h$ 's are

$$
\left\{\begin{array} { l l } 
{ h _ { 1 } = 3 / 4 + \pi / 2 0 + 2 y + \varepsilon , } & { h _ { 2 } = h _ { 1 } + x , } \\
{ h _ { 3 } = 3 / 8 + x + y + h _ { 1 } + \varepsilon , } & { h _ { 4 } = y , }
\end{array} \quad \text { with } \quad \left\{\begin{array}{l}
x=0.0752 \\
y=0.0268
\end{array}\right.\right.
$$

Thus by (3.20) we derive the last inequality for $N^{*}(\alpha, Q, C)$ in Lemma 3.1.

(v) We discuss the remaining case in which $0.504<\lambda \leq 0.696$. By [H-B, Theorem 2] we know that there are at most two zeros of the function $\prod_{\chi(\bmod q)} L(s, \chi)$ for any fixed $q \leq Q$ in the given $D$ (in $(3.1)$ ). Hence completely similar to [LLW, $\S 3$, case(v)] with the use of [Che, Lemma 4] instead of [G2, Lemma 9] there we can obtain

$$
N^{*}(\alpha, Q, C) \leq \frac{(1+\varepsilon) \widetilde{M}}{2 \lambda\left(h_{2}-h_{1}\right) h_{4} \mathcal{L}}\left(e^{2 h_{3} \lambda}-\frac{e^{2 h_{2} \lambda}-e^{2 h_{1} \lambda}}{2 \lambda\left(h_{2}-h_{1}\right)}\right)
$$

where

$$
\widetilde{M}:=\max _{\chi(\bmod q), q \leq P} \max _{1 \leq j \leq 2}\left\{\frac{1}{j} \int_{\log z_{1}}^{\log z_{3}}\left|\sum_{1 \leq l \leq j} e^{-(\varrho(l, \chi)-\alpha) x}\right|^{2} d x\right\},
$$

and $\varrho(l, \chi)$ denotes the zero of $L(s, \chi)$ in $D$. The $h$ 's in (3.21) are subject to the constraints:

$$
h_{3}>h_{2}+h_{4}+3 / 8+\varepsilon \quad \text { and } \quad h_{2}>h_{1}>3 / 4+2 h_{4}+\varepsilon .
$$

We need an upper bound for $\widetilde{M}$. For any zero $\varrho(l, \chi)$ of $L(s, \chi)$ in $D$, in view of $\operatorname{Re} \varrho(l, \chi) \geq \alpha$, we have

$$
\int_{\log z_{1}}^{\log z_{3}}\left|e^{-(\varrho(l, \chi)-\alpha) x}\right|^{2} d x \leq\left(h_{3}-h_{1}\right) \mathcal{L}
$$

If a given $L(s, \chi)$ has two zeros $\varrho(1, \chi)$ and $\varrho(2, \chi)$ in $D$, we write

$$
\varrho(l, \chi)=1-\beta_{l, \chi} \mathcal{L}^{-1}+i \gamma_{l, \chi} \mathcal{L}^{-1}, \quad l=1,2 .
$$

Then $\left|\beta_{1, \chi}-\beta_{2, \chi}\right| \leq 0.696-0.364=0.332$, and applying $n_{5} \leq 1$ in Lemma 3.3 we get $\left|\gamma_{1, \chi}-\gamma_{2, \chi}\right| \geq 2 \cdot 1.06=2.12$. Hence 


$$
\begin{aligned}
& \frac{1}{2} \int_{\log z_{1}}^{\log z_{3}}\left|\sum_{1 \leq l \leq 2} e^{-(\varrho(l, \chi)-\alpha) x}\right|^{2} d x \\
& \quad=\frac{\mathcal{L}}{2} \int_{h_{1}}^{h_{3}}\left|\sum_{1 \leq l \leq 2} e^{-\left(\lambda-\beta_{l, \chi}+i \gamma_{l, \chi}\right) x}\right|^{2} d x \\
& \leq \frac{\mathcal{L}}{2} \max _{\substack{0 \leq x \leq 0.332 \\
y \geq 2.12}} \int_{h_{1}}^{h_{3}}\left|1+e^{-(x+i y) t}\right|^{2} d t \\
& \leq \frac{\mathcal{L}}{2} \max _{\substack{0 \leq x \leq 0.332 \\
y \geq 2.12}} \int_{h_{1}}^{h_{3}}\left(1+2 e^{-x t} \cos y t+e^{-2 x t}\right) d t .
\end{aligned}
$$

Recall (3.23); numerical experiments show that the optimal choices of $h$ 's are approximately

$$
\left\{\begin{array}{l}
h_{1}=3 / 4+2 v+\varepsilon, \quad h_{2}=3 / 4+u+2 v+\varepsilon \\
h_{3}=3 / 4+3 / 8+u+3 v+2 \varepsilon, \quad h_{4}=v
\end{array}\right.
$$

with

$$
\left\{\begin{array}{l}
u=0.417 \\
v=0.205
\end{array}\right.
$$

With the above choices of $h_{1}$ and $h_{3},(3.25)$ can be estimated as

$$
\leq 1.516 \mathcal{L}
$$

directly by the "Mathematica software". From (3.22) and (3.24) to (3.27) we can summarize that

$$
\widetilde{M} \leq 1.516 \mathcal{L} .
$$

From $(3.21),(3.26)$ and $(3.28)$ we get the first inequality for $N^{*}(\alpha, Q, C)$ in Lemma 3.1. The proof of Lemma 3.1 is thus complete.

4. The circle method. From now on, we let $N$ be a sufficiently large positive number, and let

$$
\theta:=1 /\left(15-11 \varepsilon_{1}\right), \quad Q:=N^{\theta}, \quad T:=Q^{3}, \quad \tau:=N^{-1} Q^{1+\varepsilon_{1}},
$$

where $\varepsilon_{1}$ is a fixed sufficiently small positive number. For $1 \leq j \leq 3$, let

$$
N_{j}:=N\left|a_{j}\right|^{-1}, \quad N_{j}^{\prime}:=N\left(4\left|a_{j}\right|\right)^{-1} .
$$

Put

$$
A:=\max \left\{\left|a_{1}\right|,\left|a_{2}\right|,\left|a_{3}\right|\right\} .
$$

We always assume

$$
A^{3+2 \varepsilon_{1}} \ll Q .
$$


Denote by $\Lambda(n)$ the von Mangoldt function, and define, for any real $y$, $e(y):=\exp (i 2 \pi y)$

$$
S_{j}(y):=\sum_{N_{j}^{\prime}<n \leq N_{j}} \Lambda(n) e\left(a_{j} n y\right)
$$

and

$$
S_{j}(\chi, y):=\sum_{N_{j}^{\prime}<n \leq N_{j}} \Lambda(n) \chi(n) e\left(a_{j} n y\right),
$$

where $\chi$ is modulo $q \leq Q$. Put

$$
I(b):=\sum_{\left(n_{1}, n_{2}, n_{3}\right)} \Lambda\left(n_{1}\right) \Lambda\left(n_{2}\right) \Lambda\left(n_{3}\right)
$$

where $\sum_{\left(n_{1}, n_{2}, n_{3}\right)}$ denotes the summation over all triples $\left(n_{1}, n_{2}, n_{3}\right)$ satisfying $\sum_{1 \leq j \leq 3} a_{j} n_{j}=b$ and $N_{j}^{\prime}<n_{j} \leq N_{j}, 1 \leq j \leq 3$. For any integers $h$ and $q$ with $1 \leq h \leq q \leq Q$ and $(h, q)=1$, let $m(h, q)$ be the interval $[(h-\tau) / q,(h+\tau) / q]$. Let $\mathcal{M}$ be the union of these mutually disjoint intervals and $\mathcal{M}^{\prime}$ be the complement of $\mathcal{M}$ in $[\tau, 1+\tau]$. From (4.5) and (4.6) we get

$$
I(b)=\left\{\int_{\mathcal{M}}+\int_{\mathcal{M}^{\prime}}\right\} e(-b x) \prod_{j=1}^{3} S_{j}(x) d x=: I_{1}(b)+I_{2}(b), \quad \text { say, }
$$

where $I_{1}(b)$ and $I_{2}(b)$ are the integrals on $\mathcal{M}$ and $\mathcal{M}^{\prime}$ respectively. For any integer $n$ and any character $\chi(\bmod q)$, denote the Gaussian sum by

$$
G(n, \chi):=\sum_{l=1}^{q} \chi(l) e\left(\frac{n}{q} l\right) \text { and let } G(n, q):=G\left(n, \chi_{0}\right) .
$$

If $x \in m(h, q)$, write

$$
x=h / q+\eta, \quad(h, q)=1, \quad|\eta| \leq \tau q^{-1} .
$$

By the orthogonality relation of characters, we have [D, p. 147, (2)] for $1 \leq j \leq 3$,

$$
S_{j}(x)=\varphi(q)^{-1} \sum_{\chi(\bmod q)} G\left(a_{j} h, \bar{\chi}\right) S_{j}(\chi, \eta)+O\left(L^{2}\right) .
$$

Here, and from now on, we put $L:=\log N$. The purpose of this section is to give a simplified form for $I_{1}(b)$ (see Lemma 4.7 below). To do this, we first give some preliminaries. Note that the following Lemmas 4.1 and 4.3 are essentially Lemmas 3.1 and 3.2 in [LT1] respectively. 
Lemma 4.1. For any real $y$ and any $\chi(\bmod q)$ with $q \leq Q$, let, for $1 \leq j \leq 3$,

$$
\left\{\begin{array}{l}
I_{j}(y):=\int_{N_{j}^{\prime}}^{N_{j}} e\left(a_{j} x y\right) d x, \quad \widetilde{I}_{j}(y):=\int_{N_{j}^{\prime}}^{N_{j}} x^{\widetilde{\beta}-1} e\left(a_{j} x y\right) d x \\
I_{j}(\chi, y):=\int_{N_{j}^{\prime}}^{N_{j}} e\left(a_{j} x y\right) \sum_{|\gamma| \leq T}^{\prime} x^{\varrho-1} d x
\end{array}\right.
$$

and

$$
\delta^{\prime}(\chi):=\left\{\begin{array}{ll}
1 & \text { if } \chi=\chi_{0}, \\
0 & \text { otherwise, }
\end{array} \quad \delta(\chi):= \begin{cases}1 & \text { if } \chi=\tilde{\chi} \chi_{0}, \\
0 & \text { otherwise },\end{cases}\right.
$$

where $\widetilde{\beta}$ is the possible Siegel zero in Proposition 2.3 and $\widetilde{\chi}(\bmod \widetilde{r})$ is the corresponding character, $\sum_{|\gamma| \leq T}^{\prime}$ denotes the summation over all zeros $\varrho=$ $\beta+i \gamma$ of $L(s, \chi)$ satisfying $\varrho \neq \widetilde{\beta},|\gamma| \leq T$ and $1 / 2 \leq \beta \leq 1$. Then

$$
S_{j}(\chi, y)=\delta^{\prime}(\chi) I_{j}(y)-\delta(\chi) \widetilde{I}_{j}(y)-I_{j}(\chi, y)+O\left((1+N|y|) N_{j} T^{-1} L^{2}\right) .
$$

LEMMA 4.2. Under the notations of Lemma 4.1, put

$$
\mathcal{G}_{j}(h, q, \eta):=\sum_{\chi(\bmod q)} G\left(a_{j} h, \bar{\chi}\right) I_{j}(\chi, \eta)
$$

and

$$
H_{j}(h, q, \eta):=G\left(a_{j} h, q\right) I_{j}(\eta)-\delta(q) G\left(a_{j} h, \widetilde{\chi} \chi_{0}\right) \widetilde{I}_{j}(\eta)-\mathcal{G}_{j}(h, q, \eta),
$$
where $\delta(q)=1$ if $\widetilde{r} \mid q$ and $\delta(q)=0$ otherwise. Then for any $x \in m(h, q)$,

$$
S_{j}(x)=\varphi(q)^{-1} H_{j}(h, q, \eta)+O\left((1+N|\eta|) N_{j} q^{1 / 2} T^{-1} L^{2}\right) .
$$

P r o of. This is a simple consequence of (4.8) and Lemma 4.1.

Lemma 4.3. Let $\varrho=\beta+i \gamma$ be any complex number satisfying $1 / 2 \leq \beta \leq$ 1. Then for any real $y$ we have

$$
\int_{N / 4}^{N} x^{\varrho-1} e(x y) d x \ll \begin{cases}\min \left\{N^{\beta},|y|^{-\beta}\right\} & \text { if }|\gamma| \ll 1, \\ N^{\beta}|\gamma|^{-1} & \text { if }|y| \leq|\gamma| /(4 \pi N), \\ N^{\beta}|\gamma|^{-1 / 2} & \text { if }|\gamma| /(4 \pi N)<|y| \leq 4|\gamma| /(\pi N), \\ N^{\beta-1}|y|^{-1} & \text { if }|y|>4|\gamma| /(\pi N) .\end{cases}
$$

Lemma 4.4. For any $x \geq 1$ and $y \geq 2$, let

$$
N(\alpha, x, y):=\sum_{q \leq x} \sum_{\chi(\bmod q)}^{*} \sum_{\substack{\varrho=\beta+i \gamma \\|\gamma| \leq y \\ \beta \geq \alpha}} 1,
$$

where $\varrho=\beta+i \gamma$ is any non-trivial zero of $L(s, \chi)$. Then

$$
N(\alpha, x, y) \ll\left(x^{2} y\right)^{3(1-\alpha) /(2-\alpha)} \log ^{9}\left(x^{2} y\right) \quad \text { for } 1 / 2 \leq \alpha \leq 4 / 5,
$$


and for any $\varepsilon>0$,

$$
N(\alpha, x, y) \ll\left(x^{2} y\right)^{(2+\varepsilon)(1-\alpha)} \quad \text { for } 4 / 5 \leq \alpha \leq 1 .
$$

Proof. (4.12) is [PP, Theorem 4.4], and (4.13) is [J, Theorem 1].

LEMMA 4.5. Under the notations and conditions of (4.1) to (4.4), we have for $1 \leq j \leq 3$,

$$
\Sigma_{1}:=\sum_{q \leq Q} \sum_{\chi(\bmod q)}^{*} \sum_{|\gamma| \leq T} N_{j}^{\beta-1} \ll 1 .
$$

Proof. We have

$$
\begin{aligned}
\Sigma_{1} & =-\int_{1 / 2}^{1} N_{j}^{\alpha-1} d N(\alpha, Q, T) \\
& =N_{j}^{-1 / 2} N(1 / 2, Q, T)+\left\{\int_{1 / 2}^{4 / 5}+\int_{4 / 5}^{1}\right\} N(\alpha, Q, T) N_{j}^{\alpha-1} \log N_{j} d \alpha .
\end{aligned}
$$

In view of $Q \geq A^{3}$ (in (4.4)) and $\theta=1 /\left(15-11 \varepsilon_{1}\right)$, we have for $1 \leq j \leq 3$,

$$
N_{j} \geq Q^{15-11 \varepsilon_{1}-1 / 3}
$$

By (4.12), (4.15) and $T=Q^{3}$ (in (4.1)), the first term on the right of (4.14) is $\ll Q^{-(1 / 2)\left(15-11 \varepsilon_{1}-1 / 3\right)} Q^{2} T L^{9} \ll Q^{-2}$, and the first integral on the right of $(4.14)$ is

$$
\begin{aligned}
& \ll \int_{1 / 2}^{4 / 5}\left(Q^{2} T\right)^{3(1-\alpha) /(2-\alpha)} N_{j}^{\alpha-1} L^{10} d \alpha \\
& \ll L^{10} \int_{1 / 2}^{4 / 5} Q^{\left(15-11 \varepsilon_{1}-1 / 3-15 /(2-\alpha)\right)(\alpha-1)} d \alpha \ll Q^{-0.4} .
\end{aligned}
$$

By (4.13) and (4.15), the last integral on the right of (4.14) is

$$
\begin{aligned}
& \ll \int_{4 / 5}^{1} Q^{\left(15-11 \varepsilon_{1}-1 / 3\right)(\alpha-1)}\left(Q^{2} T\right)^{(2+\varepsilon)(1-\alpha)} L d \alpha \\
& \ll \int_{4 / 5}^{1} Q^{\left(15-11 \varepsilon_{1}-1 / 3-5(2+\varepsilon)\right)(\alpha-1)} L d \alpha \ll 1 .
\end{aligned}
$$

Combining all the above, the proof of Lemma 4.5 is complete.

Lemma 4.6. Under the conditions of Lemma 4.5, for $1 \leq j \leq 3$ we have $S_{1, j}:=\sum_{q \leq Q} \sum_{\chi(\bmod q)}^{*} \sum_{|\gamma| \leq T}\left(\int_{-\tau / q}^{\tau / q}\left|\int_{N_{j}^{\prime}}^{N_{j}} x^{\varrho-1} e\left(a_{j} x \eta\right) d x\right|^{2} d \eta\right)^{1 / 2} \ll N^{1 / 2}\left|a_{j}\right|^{-1}$. 
Proof. The above integral with respect to $\eta$ is

$$
\begin{aligned}
& =\int_{-\tau / q}^{\tau / q}\left|\int_{N / 4}^{N}\left(\frac{x}{\left|a_{j}\right|}\right)^{\varrho-1} e\left(\frac{a_{j}}{\left|a_{j}\right|} x \eta\right) d\left(\frac{x}{\left|a_{j}\right|}\right)\right|^{2} d \eta \\
& =\left|a_{j}\right|^{-2 \beta} \int_{-\tau / q}^{\tau / q}\left|\int_{N / 4}^{N} x^{\varrho-1} e(x \eta) d x\right|^{2} d \eta .
\end{aligned}
$$

Hence by Lemma 4.3 the innermost sum in $S_{1, j}$ is

$$
\begin{aligned}
& \ll \sum_{|\gamma| \leq 1}\left|a_{j}\right|^{-\beta}\left\{\int_{-\tau / q}^{\tau / q} \min \left\{N^{2 \beta},|\eta|^{-2 \beta}\right\} d \eta\right\}^{1 / 2} \\
& +\sum_{1 \leq|\gamma| \leq T}\left|a_{j}\right|^{-\beta}\left\{\int_{\substack{\eta \in[0, \tau / q] \\
\eta>4|\gamma| /(\pi N)}}\left(N^{\beta-1} \eta^{-1}\right)^{2} d \eta\right. \\
& \left.+\int_{\substack{\eta \in[0, \tau / q] \\
4|\gamma| /(\pi N) \geq \eta>|\gamma| /(4 \pi N)}}\left(N^{\beta}|\gamma|^{-1 / 2}\right)^{2} d \eta+\int_{\substack{\eta \in[0, \tau / q] \\
\eta \leq|\gamma| /(4 \pi N)}}\left(N^{\beta}|\gamma|^{-1}\right)^{2} d \eta\right\}^{1 / 2} \\
& \ll N^{1 / 2}\left|a_{j}\right|^{-1} \sum_{|\gamma| \leq T} N_{j}^{\beta-1},
\end{aligned}
$$

by noting (4.2). Thus by Lemma 4.5 we get the desired result.

The following lemma is the desired simplified form for $I_{1}(b)$.

Lemma 4.7. Let $I_{1}(b)$ be defined as in (4.7). Under the assumptions of Lemma 4.5, we have

$$
I_{1}(b)=\sum_{q \leq Q} \varphi(q)^{-3} \sum_{h=1}^{q} e\left(-\frac{b}{q} h\right) \int_{-\tau / q}^{\tau / q} e(-b \eta) \prod_{j=1}^{3} H_{j}(h, q, \eta) d \eta+O\left(\Omega_{1}\right),
$$

where $\sum_{h=1}^{\prime q}$ is the summation over all $1 \leq h \leq q,(h, q)=1$, and

$$
\Omega_{1}:=N^{2} Q^{2.5+\varepsilon_{1}}\left|a_{1} a_{2} a_{3}\right|^{-1} T^{-1} L^{2} .
$$

Proof. Recalling the definition in (4.7) we have

$$
I_{1}(b)=\sum_{q \leq Q} \sum_{h=1}^{q} \int_{-\tau / q}^{\tau / q} e\left(-b\left(h q^{-1}+\eta\right)\right) \prod_{j=1}^{3} S_{j}\left(h q^{-1}+\eta\right) d \eta .
$$

We approximate $S_{j}\left(h q^{-1}+\eta\right)$ by the formulas in Lemma 4.2. So we rewrite $S_{j}\left(h q^{-1}+\eta\right)$ as $S_{j}\left(h q^{-1}+\eta\right)=B_{j}+O\left(F_{j}\right)$, where $B_{j}:=\varphi(q)^{-1} H_{j}(h, q, \eta)$ and $F_{j}:=(1+N|\eta|) N_{j} q^{1 / 2} T^{-1} L^{2}$. Firstly, we replace $S_{3}\left(h q^{-1}+\eta\right)$ in (4.17) by $B_{3}+O\left(F_{3}\right)$. After such substitution, there is an error term in (4.17) due to the term $O\left(F_{3}\right)$. In view of the definition of $F_{3}$, and then by (4.1), (4.2), 
(4.5) and (4.16), the error term is $\ll N^{2} Q^{1+\varepsilon_{1}} T^{-1}\left|a_{1} a_{2} a_{3}\right|^{-1 / 2} L^{3} \leq \Omega_{1}$. Next, we replace $S_{j}\left(h q^{-1}+\eta\right)(j=1,2)$ by $B_{j}+O\left(F_{j}\right)$. Consequently, we have

$$
\begin{aligned}
I_{1}(b)= & \sum_{q \leq Q} \sum_{h=1}^{q} \int_{-\tau / q}^{\tau / q} e\left(-b\left(h q^{-1}+\eta\right)\right) \varphi(q)^{-1} H_{3}(h, q, \eta) \\
& \times\left\{B_{1} B_{2}+O\left(\left|B_{1}\right| F_{2}+\left|B_{2}\right| F_{1}+F_{1} F_{2}\right)\right\} d \eta+O\left(\Omega_{1}\right) .
\end{aligned}
$$

Denote by $E_{1}, E_{2}$ and $E_{3}$ the total error induced by $\left|B_{1}\right| F_{2},\left|B_{2}\right| F_{1}$ and $F_{1} F_{2}$ in (4.18) respectively. Then they can be estimated in precisely the same way; and the most difficult case is that for $E_{2}$ (or $E_{1}$ ). So we only give the details of the estimate for $E_{2}$ to illustrate the methods. We have

$$
\begin{aligned}
E_{2} \ll & N_{1} Q^{1+\varepsilon_{1}} T^{-1} L^{2} \\
& \times \sum_{q \leq Q} q^{-1 / 2} \varphi(q)^{-2} \sum_{h=1}^{q} \int_{-\tau / q}^{\tau / q}\left|H_{2}(h, q, \eta) H_{3}(h, q, \eta)\right| d \eta .
\end{aligned}
$$

From (4.11), we have for $1 \leq j \leq 3$,

$$
\begin{aligned}
\left|H_{j}(h, q, \eta)\right| \leq & \left|G\left(a_{j} h, q\right) I_{j}(\eta)\right|+\delta(q)\left|G\left(a_{j} h, \widetilde{\chi} \chi_{0}\right) \widetilde{I}_{j}(\eta)\right| \\
& +\left|\mathcal{G}_{j}(h, q, \eta)\right| .
\end{aligned}
$$

By (4.20), multiplying out $\left|H_{2}(h, q, \eta) H_{3}(h, q, \eta)\right|$, we get 9 terms. The contribution of these terms to (4.19) can be estimated in the same way. So we only give the details of the estimation of the typical terms corresponding to $\left|\mathcal{G}_{2}(h, q, \eta) \mathcal{G}_{3}(h, q, \eta)\right|,\left|\prod_{j=2}^{3} G\left(a_{j} h, q\right) I_{j}(\eta)\right|, \delta(q) \mid G\left(a_{2} h, q\right) I_{2}(\eta) \times$ $G\left(a_{3} h, \widetilde{\chi} \chi_{0}\right) \widetilde{I}_{3}(\eta) \mid$ and $\delta(q)\left|G\left(a_{2} h, \widetilde{\chi} \chi_{0}\right) \widetilde{I}_{2}(\eta) \mathcal{G}_{3}(h, q, \eta)\right|$. Denote by $E_{21}$, $E_{22}, E_{23}$ and $E_{24}$ the total error to (4.19) induced by them respectively. In view of (4.9) and (4.10), we get

$$
\begin{aligned}
E_{21} \ll & N_{1} Q^{1+\varepsilon_{1}} T^{-1} L^{2} \\
& \times \sum_{r_{2} \leq Q} \sum_{\chi_{2}\left(\bmod r_{2}\right)}^{*} \sum_{\left|\gamma_{2}\right| \leq T}^{\prime} \sum_{r_{3} \leq Q} \sum_{\chi_{3}\left(\bmod r_{3}\right)}^{*} \sum_{\left|\gamma_{3}\right| \leq T}^{\prime} \sum_{\substack{q \leq Q \\
\left[r_{2}, r_{3}\right] \mid q}} q^{-1 / 2} \varphi(q)^{-2} \\
& \times \sum_{h=1}^{q}\left|G\left(a_{2} h, \bar{\chi}_{2} \chi_{0}\right) G\left(a_{3} h, \bar{\chi}_{3} \chi_{0}\right)\right| \int_{-\tau / q}^{\tau / q}\left|\prod_{j=2}^{3} \int_{N_{j}^{\prime}}^{N_{j}} x^{\varrho_{j}-1} e\left(a_{j} x \eta\right) d x\right| d \eta .
\end{aligned}
$$

Now apply Cauchy's inequality to the integral with respect to $\eta$. Noting $r_{2}, r_{3} \leq q$, and using the trivial bound $\left|G\left(a_{j} h, \bar{\chi}_{j} \chi_{0}\right)\right| \leq \varphi(q), j=2,3$, by Lemma 4.6 we get

$$
E_{21} \ll N_{1} Q^{1+\varepsilon_{1}} T^{-1} L^{2} S_{1,2} S_{1,3} \sum_{q \leq Q} q^{-1 / 2} \varphi(q)^{-2} \varphi(q)^{3} \ll \Omega_{1} .
$$


In view of (4.9), by Lemma 4.3 , we have for $1 \leq j \leq 3$,

$$
\int_{-\tau / q}^{\tau / q}\left|\widetilde{I}_{j}(\eta)\right|^{2} d \eta \ll\left|a_{j}\right|^{-2 \widetilde{\beta}}\left\{\int_{0}^{N^{-1}} N^{2 \widetilde{\beta}} d \eta+\int_{N^{-1}}^{\tau / q} \eta^{-2 \widetilde{\beta}} d \eta\right\} \ll N\left|a_{j}\right|^{-2} .
$$

Hence, similar to the estimation of $E_{21}$, we have

$$
E_{24} \ll N_{1} Q^{1+\varepsilon_{1}} T^{-1} L^{2} S_{1,3} \sum_{q \leq Q}\left(\int_{-\tau / q}^{\tau / q}\left|\widetilde{I}_{2}(\eta)\right|^{2} d \eta\right)^{1 / 2} q^{-1 / 2} \varphi(q) \ll \Omega_{1} .
$$

Similarly, $E_{22}, E_{23} \ll \Omega_{1}$. Hence $E_{2} \ll \Omega_{1}$. In precisely the same way we have $E_{1}, E_{3} \ll \Omega_{1}$. Then Lemma 4.7 follows from these estimates and (4.18).

5. The estimation of $M_{1}$ and $M_{3}$. Multiplying out the product $\prod_{j=1}^{3} H_{j}(h, q, \eta)$ in Lemma 4.7 , we get 27 terms (if $\widetilde{\beta}$ exists). They are grouped into the following three categories:

- $\mathcal{J}_{1}$ : the term $\prod_{j=1}^{3}\left\{G\left(a_{j} h, q\right) I_{j}(\eta)\right\}$

- $\mathcal{J}_{2}: 19$ terms (if $\widetilde{\beta}$ exists), each has at least one $\mathcal{G}_{j}(h, q, \eta)$ as factor;

- $\mathcal{J}_{3}$ : the 7 terms remaining (if $\widetilde{\beta}$ exists).

For $i=1,2,3$, define

$$
\begin{aligned}
M_{i}:= & \sum_{q \leq Q} \varphi(q)^{-3} \sum_{h=1}^{q} e\left(-\frac{b}{q} h\right) \\
& \times \int_{-\tau / q}^{\tau / q} e(-b \eta)\left\{\text { sum of the terms in } \mathcal{J}_{i}\right\} d \eta .
\end{aligned}
$$

Then by Lemma 4.7 we get

$$
I_{1}(b)=M_{1}+M_{2}+M_{3}+O\left(\Omega_{1}\right),
$$

if one assumes (4.4). For the estimation of $M_{1}$ and $M_{3}$, we need the following notations. For any positive integer $q$, define

$$
A(q):=\varphi(q)^{-3} \sum_{h=1}^{q} e\left(-\frac{b}{q} h\right) \prod_{j=1}^{3} G\left(a_{j} h, q\right) .
$$

By [LT1, Lemma 4.1] $A(q)$ is multiplicative. For any prime $p$, put

$$
s(p):=1+A(p) .
$$

Let $r_{1}, r_{2}, r_{3}$ be any positive integers and denote by $\left[r_{1}, r_{2}, r_{3}\right]$ the least common multiple of $r_{1}, r_{2}$ and $r_{3}$. For any primitive characters $\chi_{j}\left(\bmod r_{j}\right)$ 
$(1 \leq j \leq 3)$ and $\left[r_{1}, r_{2}, r_{3}\right] \mid q$, define

$$
Z(q):=Z\left(q ; \chi_{1}, \chi_{2}, \chi_{3}\right):=\sum_{h=1}^{q} e\left(-\frac{b}{q} h\right) \prod_{j=1}^{3} G\left(a_{j} h, \chi_{j} \chi_{0}\right),
$$

where $\chi_{0}$ is the principal character modulo $q$. For abbreviation, we let $\sum_{\sim}$ denote the summation over $\left(l_{1}, l_{2}, l_{3}\right)$ satisfying

$$
1 \leq l_{j} \leq \widetilde{r}, \quad\left(l_{j}, \widetilde{r}\right)=1, \quad 1 \leq j \leq 3 \quad \text { and } \quad \sum_{j=1}^{3} a_{j} l_{j} \equiv b(\bmod \widetilde{r}) .
$$

For $1 \leq m_{1} \leq m_{2} \leq \ldots \leq 3$, put

$$
G\left(m_{1}, m_{2}, \ldots\right):=\sum_{\sim} \widetilde{\chi}\left(l_{m_{1}}\right) \widetilde{\chi}\left(l_{m_{2}}\right) \ldots
$$

and

$$
P\left(m_{1}, m_{2}, \ldots\right):=\int_{\mathcal{D}}\left(N x_{m_{1}}\right)^{\widetilde{\beta}-1}\left(N x_{m_{2}}\right)^{\widetilde{\beta}-1} \ldots d x_{1} d x_{2}
$$

where

$$
\mathcal{D}:=\left\{\left(x_{1}, x_{2}\right):\left(4\left|a_{j}\right|\right)^{-1} \leq x_{j} \leq\left|a_{j}\right|^{-1}, j=1,2,3\right\},
$$

and $x_{3}=a_{3}^{-1}\left(b N^{-1}-a_{1} x_{1}-a_{2} x_{2}\right)$.

Lemma 5.1. For any complex numbers $\varrho_{j}$ with $0<\operatorname{Re} \varrho_{j} \leq 1,1 \leq j \leq 3$, we have

$$
\begin{aligned}
\int_{-\infty}^{\infty}\left\{\prod_{j=1}^{3} \int_{N_{j}^{\prime}}^{N_{j}} x^{\varrho_{j}-1} e\left(a_{j} x \eta\right) d x\right\} e(-b \eta) d \eta & \\
& =N^{2}\left|a_{3}\right|^{-1} \int_{\mathcal{D}} \prod_{j=1}^{3}\left(N x_{j}\right)^{\varrho_{j}-1} d x_{1} d x_{2}
\end{aligned}
$$

where $\mathcal{D}$ is defined as in (5.6).

Proof. The lemma can be proved by precisely the same way as that of [LT1, Lemma 4.7].

Lemma 5.2. Let $Z(q)$ and $s(p)$ be defined as in (5.5) and (5.4) respectively, and let $r=\left[r_{1}, r_{2}, r_{3}\right]$. Then under the conditions of (1.2) and (1.3), we have

$$
\prod_{p} s(p) \gg 1
$$

and

$$
\sum_{\substack{q \leq Q \\ r \mid q}} \varphi(q)^{-3}|Z(q)| \leq 2.140782 \prod_{p} s(p)
$$


P roof. (5.7) is [LT1, Lemma 4.4(2)]. We only need to prove (5.8). Completely similar to [LT1, Lemma 4.6] we have

$$
\sum_{\substack{q \leq Q \\ r \mid q}} \varphi(q)^{-3}|Z(q)| \leq \prod_{p \mid r} s(p) \prod_{p \nmid r}(1+|A(p)|)=\prod_{p \nmid r} \frac{1+|A(p)|}{1+A(p)} \prod_{p} s(p) .
$$

Note that (1.2) and (1.3) imply $A(2)=1$. For any prime $p \geq 3$, the proof of [LT1, Lemma 4.2] shows that either $|A(p)| \leq(p-1)^{-2}$ or $A(p)>0$ under the conditions (1.2) and (1.3). Thus

$$
\prod_{p \nmid r} \frac{1+|A(p)|}{1+A(p)} \leq \prod_{p \geq 3} \frac{1+(p-1)^{-2}}{1-(p-1)^{-2}}=\prod_{p \geq 3}\left(1+\frac{2}{p(p-2)}\right) \leq 2.140782 .
$$

The proof of Lemma 5.2 is complete.

Lemma 5.3. Let $M_{1}$ be defined as in (5.1). Then

$$
M_{1}=M_{0}+O\left(\Omega_{2}\right)
$$

where

$$
M_{0}:=N^{2}\left|a_{3}\right|^{-1}\left(\prod_{p} s(p)\right) \int_{\mathcal{D}} d x_{1} d x_{2}
$$

and

$$
\Omega_{2}:=N^{2} Q^{-1+\varepsilon_{1} / 4}\left|a_{1} a_{2} a_{3}\right|^{-1} .
$$

P r o of. By the use of [LT1, Lemma 4.4(4) with $B$ replaced by $B^{\varepsilon}$ ] (note that the proof in [LT1, Lemma 4.4(4)] actually allows us to replace the $B$ there by $B^{1 / \log \log B}$ ), the lemma can be proved in the same way as [LT1, Lemma 5.1] with a more careful estimate of bounds in terms of $a_{j}$ 's rather than $B$.

Lemma 5.4. Let $M_{3}$ be defined as in (5.1). Then

$$
\begin{aligned}
M_{3}= & N^{2}\left|a_{3}\right|^{-1} \widetilde{r} \varphi(\widetilde{r})^{-3}\left(\prod_{p \nmid \widetilde{r}} s(p)\right)\left\{-\sum_{j=1}^{3} G(j) P(j)+\sum_{1 \leq i<j \leq 3} G(i, j) P(i, j)\right. \\
& -G(1,2,3) P(1,2,3)\}+O\left(\widetilde{r} \Omega_{2}+N^{2} Q^{-1-\varepsilon_{1}}\right) .
\end{aligned}
$$

Proof. The lemma can be proved in the same way as [LT1, Lemma 5.2] with a more careful estimate of bounds in terms of $a_{j}$ 's rather than $B$.

Lemma 5.5. If the exceptional zero $\widetilde{\beta}$ defined as in Proposition 2.3 exists, put

$$
\omega:=(1-\widetilde{\beta}) \mathcal{L} .
$$

Then $M_{1}+M_{3} \geq 20 \omega^{3} M_{0}+O\left(\widetilde{r} \Omega_{2}+N^{2} Q^{-1-\varepsilon_{1}}\right)$. 
Proof. The combination of Lemmas 5.3 and 5.4 together with (5.10) and [LT1, (4.16)] gives

$$
\begin{aligned}
= & M_{0}+N^{2}\left|a_{3}\right|^{-1} \widetilde{r} \varphi(\widetilde{r})^{-3}\left(\prod_{p \nmid \widetilde{r}} s(p)\right)\left\{-\sum_{j=1}^{3} G(j) P(j)\right. \\
& \left.+\sum_{1 \leq i<j \leq 3} G(i, j) P(i, j)-G(1,2,3) P(1,2,3)\right\}+O\left(\widetilde{r} \Omega_{2}+N^{2} Q^{-1-\varepsilon_{1}}\right) \\
= & N^{2}\left|a_{3}\right|^{-1} \widetilde{r} \varphi(\widetilde{r})^{-3}\left(\prod_{p \nmid \widetilde{r}} s(p)\right) \sum_{\sim} \int_{\mathcal{D}} \prod_{j=1}^{3}\left(1-\widetilde{\chi}\left(l_{j}\right)\left(N x_{j}\right)^{\widetilde{\beta}-1}\right) d x_{1} d x_{2} \\
& +O\left(\widetilde{r} \Omega_{2}+N^{2} Q^{-1-\varepsilon_{1}}\right) .
\end{aligned}
$$

Since, by (4.2) and (5.6), $N x_{j} \geq N_{j}^{\prime}(1 \leq j \leq 3)$, the last summation $\sum_{\sim}$ in (5.13) is

$$
\begin{aligned}
& \geq \sum_{\sim} \int_{\mathcal{D}} \prod_{j=1}^{3}\left(1-\left(N x_{j}\right)^{\widetilde{\beta}-1}\right) d x_{1} d x_{2} \\
& \geq\left(\sum_{\sim} \int_{\mathcal{D}} d x_{1} d x_{2}\right) \prod_{j=1}^{3}\left(1-N_{j}^{\prime \widetilde{\beta}-1}\right) .
\end{aligned}
$$

Furthermore, in view of (2.13) we have $\omega \leq 0.364$. Thus noting $N_{j}^{\prime} \geq$ $4^{-1} Q^{15-11 \varepsilon_{1}-1 / 3}$ we have for $1 \leq j \leq 3$,

$$
\begin{aligned}
1-N_{j}^{\prime \widetilde{\beta}-1} & \geq 1-\left(4^{-1} Q^{15-11 \varepsilon_{1}-1 / 3}\right)^{\widetilde{\beta}-1} \\
& =1-\exp \left\{(\widetilde{\beta}-1) \log \left(4^{-1} Q^{15-11 \varepsilon_{1}-1 / 3}\right)\right\} \\
& \geq 1-\exp \left\{\left(15-12 \varepsilon_{1}-1 / 3\right)(\widetilde{\beta}-1) \mathcal{L}\right\} \\
& \geq 1-\exp \left\{-\left(15-12 \varepsilon_{1}-1 / 3\right) \omega\right\} \\
& \geq \frac{1-\exp \left\{-0.364\left(15-12 \varepsilon_{1}-1 / 3\right)\right\}}{0.364} \omega \geq 2.734 \omega
\end{aligned}
$$

Now, substituting (5.15) into (5.14), then into (5.13), and noting (5.10) and $[\mathrm{LT} 1,(4.16)]$, one can easily derive the desired result. The proof of Lemma 5.5 is complete.

LEMMA 5.6. If $\widetilde{\beta}$ exists, we have

$$
M_{1}+M_{3}=M_{0}+O\left(\Omega_{2}+N^{2} \widetilde{r}^{-1} \log ^{3} L+N^{2} Q^{-1-\varepsilon_{1}}\right) .
$$

Proof. This can be proved in the same way as [LT1, Lemma 5.5]. 


\section{Further estimates on triple sums}

LEMmA 6.1. Let $\varepsilon_{2}$ be a fixed sufficiently small positive constant and $Q>K\left(\varepsilon_{2}\right)$ which is a positive constant depending on $\varepsilon_{2}$ only. Suppose that the exceptional zero $\widetilde{\beta}$ exists and satisfies $\omega \leq \varepsilon_{2}$. Then for $1 \leq j \leq 3$,

$$
\Sigma_{2}:=\sum_{q \leq Q} \sum_{\chi(\bmod q)}^{*} \sum_{|\gamma| \leq Q^{1+\varepsilon_{1}} q^{-1}}^{\prime} N_{j}^{\beta-1} \ll \varepsilon_{2}^{1 / 2} \omega^{3} .
$$

Proof. For any zero $\varrho=\beta+i \gamma$ of $L(s, \chi)$ with $|\gamma| \leq Q^{1+\varepsilon_{1}} q^{-1}$ and $\varrho \neq \widetilde{\beta}$, by $(2.12)$ and (5.12) we have for any parameter $0<c<1$,

$$
\begin{aligned}
\beta & \leq 1-\min \left\{\frac{c}{6}, \frac{(1-c)(2 / 3-\varepsilon)}{\log \left([q, \widetilde{r}] Q^{1+\varepsilon_{1}} q^{-1}\right)} \log \left(\frac{(1-c)(2 / 3-\varepsilon)}{(1-\widetilde{\beta}) \log \left([q, \widetilde{r}] Q^{1+\varepsilon_{1}} q^{-1}\right)}\right)\right\} \\
& \leq 1-\min \left\{\frac{c}{6}, \frac{(1-c)(2 / 3-\varepsilon)}{\left(2+\varepsilon_{1}\right) \mathcal{L}} \log \left(\frac{(1-c)(2 / 3-\varepsilon)}{\left(2+\varepsilon_{1}\right) \omega}\right)\right\} .
\end{aligned}
$$

In view of (2.13) and (5.12), we have for any positive $\varepsilon$,

$$
\omega \geq Q^{-\varepsilon}
$$

providing that $Q \geq K(\varepsilon)$ which is a positive constant depending on $\varepsilon$ only. Hence, for any fixed sufficiently small parameter $c$, the second term inside the last curly brackets in (6.1) is always smaller than the first one $c / 6$. Thus the above ensures

$$
\begin{aligned}
\beta & \leq 1-\frac{(1-c)(2 / 3-\varepsilon)}{\left(2+\varepsilon_{1}\right) \mathcal{L}} \log \left(\frac{(1-c)(2 / 3-\varepsilon)}{\left(2+\varepsilon_{1}\right) \omega}\right) \\
& \leq 1-\frac{1-\varepsilon_{3}}{3 \mathcal{L}} \log \left(\frac{1-\varepsilon_{3}}{3 \omega}\right)=: 1-\eta(Q),
\end{aligned}
$$

where $\varepsilon_{3}=\varepsilon_{3}\left(c, \varepsilon, \varepsilon_{1}\right)$ is a positive constant depending on $c, \varepsilon$ and $\varepsilon_{1}$ only. And $\varepsilon_{3}$ becomes sufficiently small if $c, \varepsilon$ and $\varepsilon_{1}$ are chosen to be sufficiently small. Hence by putting

$$
N^{*}(\alpha, Q):=\sum_{q \leq Q} \sum_{\chi(\bmod q)}^{*} \sum_{\substack{\varrho=\beta+i \gamma \neq \widetilde{\beta} \\|\gamma| \leq Q^{1+\varepsilon_{1}} q^{-1} \\ \beta \geq \alpha}} 1,
$$

we can write

$$
\begin{aligned}
\Sigma_{2}= & -\int_{1 / 2}^{1-\eta(Q)} N_{j}^{\alpha-1} d N^{*}(\alpha, Q) \\
= & N_{j}^{-1 / 2} N^{*}(1 / 2, Q) \\
& +\left\{\int_{1 / 2}^{4 / 5}+\int_{4 / 5}^{1-\eta(Q)}\right\} N^{*}(\alpha, Q) N_{j}^{\alpha-1} \log N_{j} d \alpha .
\end{aligned}
$$


Similar to the proof of Lemma 4.5, the sum of the first two terms on the right hand side of (6.5) can be estimated as $\ll Q^{-0.4} \leq \varepsilon_{2} \omega^{3}$ by noting (6.2). To estimate the last term, we first use (4.13) to bound $N^{*}(\alpha, Q)$ as follows. Let $c_{k}=1-2^{-k}$ for $k \geq 0$. So $2 c_{k}=1+c_{k-1}$ for $k \geq 1$. Let $k_{0}$ be a fixed sufficiently large integer. Then by (6.4) and (4.13) we have

$$
\begin{aligned}
N^{*}(\alpha, Q) \leq & \sum_{k=1}^{k_{0}} \sum_{Q^{c_{k}-1}<q \leq Q^{c_{k}}} \sum_{\chi(\bmod q)}^{*} \sum_{\substack{\varrho=\beta+i \gamma \\
|\gamma| \leq Q^{1-c_{k}-1+\varepsilon_{1}} \\
\beta \geq \alpha}}^{\prime} 1 \\
& +\sum_{Q^{c_{k}<q \leq Q}} \sum_{\chi(\bmod q)}^{*} \sum_{\substack{\varrho=\beta+i \gamma \\
|\gamma| \leq Q^{1-c_{k_{0}}+\varepsilon_{1}} \\
\beta \geq \alpha}}^{\prime} 1 \\
\ll & \sum_{k=1}^{k_{0}} Q^{\left(1+2 c_{k}+\varepsilon_{1}-c_{k-1}\right)(2+\varepsilon)(1-\alpha)}+Q^{\left(2+1+\varepsilon_{1}-c_{k_{0}}\right)(2+\varepsilon)(1-\alpha)} \\
\ll & \ll Q^{\left(2+2 \varepsilon_{1}\right)(2+\varepsilon)(1-\alpha)},
\end{aligned}
$$

providing that $2^{-k_{0}} \leq \varepsilon_{1}$ where the implied constant in the last $\ll$ depends on $k_{0}$. Hence by (4.15) and in view of the definition of $\eta(Q)$ in (6.3), the last integral in (6.5) can be estimated as

$$
\begin{aligned}
& \ll \int_{4 / 5}^{1-\eta(Q)} Q^{\left(15-11 \varepsilon_{1}-1 / 3-\left(2+2 \varepsilon_{1}\right)(2+\varepsilon)\right)(\alpha-1)} L d \alpha \\
& \ll \exp \left\{-\left(15-11 \varepsilon_{1}-1 / 3-\left(2+2 \varepsilon_{1}\right)(2+\varepsilon)\right) \frac{1-\varepsilon_{3}}{3} \log \left(\frac{1-\varepsilon_{3}}{3 \omega}\right)\right\} \\
& \ll \omega^{3.5} \ll \varepsilon_{2}^{1 / 2} \omega^{3} .
\end{aligned}
$$

The proof of Lemma 6.1 is complete.

LEMMA 6.2. Under the notations of Lemma 6.1, for any positive constant $C>0$ let $Q>K\left(C, \varepsilon_{2}\right)$ which is a positive constant depending on $C$ and $\varepsilon_{2}$ only. If (i) $\widetilde{\beta}$ does not exist or (ii) $\widetilde{\beta}$ exists and satisfies $\omega>\varepsilon_{2}$, then we have for $1 \leq j \leq 3$,

$$
\Sigma_{3}:=\sum_{q \leq Q} \sum_{\chi(\bmod q)}^{*} \sum_{|\gamma| \leq C}^{\prime} N_{j}^{\prime \beta-1} \leq \begin{cases}0.096 & \text { if } \widetilde{\beta} \text { does not exist } \\ 0.5633 \omega^{3} & \text { if } \widetilde{\beta} \text { exists. }\end{cases}
$$

P r o of. We first prove the lemma under the assumption that the exceptional zero $\widetilde{\beta}$ does not exist. By Proposition 2.3 and in view of the bounds for $\lambda$ in Lemma 3.1, we can write 


$$
\begin{aligned}
\Sigma_{3}= & N_{j}^{\prime-1 / 2} N^{*}(1 / 2, Q, C)+\left\{\int_{1 / 2}^{4 / 5}+\int_{4 / 5}^{1-\mathcal{L}^{-1} \log \log \mathcal{L}}+\int_{1-\mathcal{L}^{-1} \log \log \mathcal{L}}^{1-6 / \mathcal{L}}\right. \\
& +\int_{1-6 / \mathcal{L}}^{1-2 / \mathcal{L}}+\int_{1-2 / \mathcal{L}}^{1-1 / \mathcal{L}}+\int_{1-1 / \mathcal{L}}^{1-0.696 / \mathcal{L}}+\int_{1-0.696 / \mathcal{L}}^{1-0.504 / \mathcal{L}} \\
& \left.+\int_{1-0.504 / \mathcal{L}}^{1-0.364 / \mathcal{L}}\right\} N^{*}(\alpha, Q, C) N_{j}^{\prime \alpha-1} \log N_{j}^{\prime} d \alpha \\
= & : \sum_{l=1}^{9} C_{l}, \quad \text { say. }
\end{aligned}
$$

By (4.12) and (4.15) we have

and

$$
C_{1} \ll Q^{-\left(15-11 \varepsilon_{1}-1 / 3\right) / 2+2} L^{9} C \ll Q^{-5}
$$

$$
C_{2} \ll \int_{1 / 2}^{4 / 5} L^{10} Q^{\left(15-11 \varepsilon_{1}-1 / 3\right)(\alpha-1)}\left(Q^{2} C\right)^{3(1-\alpha) /(2-\alpha)} d \alpha \ll Q^{-1.9} .
$$

By (4.13) and (4.15) we have

$$
C_{3} \ll \int_{4 / 5}^{1-\mathcal{L}^{-1} \log \log \mathcal{L}} L Q^{\left(15-11 \varepsilon_{1}-1 / 3\right)(\alpha-1)}\left(Q^{2} C\right)^{(2+\varepsilon)(1-\alpha)} d \alpha \ll(\log \mathcal{L})^{-10}
$$

Now we use Lemma 3.1 to estimate $C_{4}$ to $C_{8}$. So we use the notation $\alpha=1-\lambda / \mathcal{L}$. In view of (4.15) and (4.2) we have

$$
\begin{aligned}
C_{4} & \leq \int_{6}^{\log \log \mathcal{L}} N^{*}(1-\lambda / \mathcal{L}, Q, C) Q^{-\left(15-12 \varepsilon_{1}-1 / 3\right) \lambda / \mathcal{L}}\left(\mathcal{L}^{-1} \log N\right) d \lambda \\
& \leq 15 \int_{6}^{\log \log \mathcal{L}} E\left(\varepsilon_{1}, \lambda\right) N_{4}^{*} d \lambda \leq 7 \cdot 10^{-29},
\end{aligned}
$$

where $E\left(\varepsilon_{1}, \lambda\right):=\exp \left(-\left(15-12 \varepsilon_{1}-1 / 3\right) \lambda\right)$. Similarly we have

$$
C_{5} \leq 8.7 \cdot 10^{-9} ; \quad C_{6} \leq 0.0008682 ; \quad C_{7} \leq 0.02361 ; \quad C_{8} \leq 0.062157 .
$$

For $C_{9}$, by Lemma 2.5 we know that if $1-0.504 / \mathcal{L} \leq \alpha \leq 1-0.364 / \mathcal{L}$, then $N^{*}(\alpha, Q, C) \leq 2$, whence by $(4.15)$,

$$
C_{9} \leq 2 \cdot 15 \int_{0.364}^{0.504} E\left(\varepsilon_{1}, \lambda\right) d \lambda \leq 0.008564
$$

Combining all the above, we get under the assumption that $\widetilde{\beta}$ does not exist,

$$
\Sigma_{3} \leq 0.096 \text {. }
$$


Now we come to estimate $\Sigma_{3}$ under the assumption that $\widetilde{\beta}$ exists. We separate the arguments into six cases according to the values of the upper bounds for $\omega$ (see (5.12)) at $10^{-5}, 0.0025,0.066,0.2,0.306$ and 0.364 .

(i) If $\omega \leq 10^{-5}$, then by noting $\omega \geq \varepsilon_{2}$, for any zero $\varrho=\beta+i \gamma(\neq \widetilde{\beta})$ of the function (2.1), we can show easily that the bound (6.3) holds by a similar (but simpler) argument as for (6.3). Hence by a similar argument to the case where $\widetilde{\beta}$ does not exist and noting (4.15), we get

$$
\begin{aligned}
\Sigma_{3} \leq & (\log \mathcal{L})^{-9} \omega^{3} \\
& +\int_{1-\mathcal{L}^{-1} \log \log \mathcal{L}}^{1-\eta(Q)} N^{*}(\alpha, Q, C) Q^{\left(15-12 \varepsilon_{1}-1 / 3\right)(\alpha-1)} \log N d \alpha
\end{aligned}
$$

To estimate the integral in (6.7), we consider two cases according to $\eta(Q) \geq$ $6 / \mathcal{L}$ or not. When $\eta(Q) \geq 6 / \mathcal{L}$, from $\left(\left(1-\varepsilon_{3}\right) /(3 \mathcal{L})\right) \log \left(\left(1-\varepsilon_{3}\right) /(3 \omega)\right) \geq 6 / \mathcal{L}$ we get $\omega \leq 3^{-1} \exp (-18-\varepsilon)$. Thus by the last inequality in Lemma 3.1, the integral in (6.7) can be estimated as

$$
\begin{aligned}
& \leq \int_{\eta(Q) \mathcal{L}}^{\log \log \mathcal{L}} 15(42.54)(1+35.385 / 6) \exp (2.87538 \lambda) Q^{-\left(15-12 \varepsilon_{1}-1 / 3\right) \lambda \mathcal{L}^{-1}} d \lambda \\
& \leq 374\left(\frac{3 \omega}{1-\varepsilon_{3}}\right)^{\left(1-\varepsilon_{3}\right)\left(15-12 \varepsilon_{1}-1 / 3-2.87538\right) / 3} \leq 374\left(\frac{3 \omega}{1-\varepsilon_{3}}\right)^{3.930428} \\
& \leq 0.0006 \omega^{3} .
\end{aligned}
$$

When $\eta(Q)<6 / \mathcal{L}$, by $\left(\left(1-\varepsilon_{3}\right) /(3 \mathcal{L})\right) \log \left(\left(1-\varepsilon_{3}\right) /(3 \omega)\right)<6 / \mathcal{L}$ we get $\omega>\left(3+\varepsilon_{3}\right)^{-1} \exp \left(-18-\varepsilon_{3}\right)$. Thus in view of $C_{4} \leq 7 \cdot 10^{-29}$, the integral in $(6.7)$ is

$$
\begin{aligned}
\leq & \frac{7 \cdot 10^{-29}}{\left(\left(3+\varepsilon_{3}\right)^{-1} \exp \left(-18-\varepsilon_{3}\right)\right)^{3}} \omega^{3} \\
& +\int_{1-6 / \mathcal{L}}^{1-\eta(Q)} N^{*}(\alpha, Q, C) Q^{\left(15-12 \varepsilon_{1}-1 / 3\right)(\alpha-1)} \log N d \alpha \\
\leq & 5.36 \cdot 10^{-4} \omega^{3}+\int_{1-6 / \mathcal{L}}^{1-\eta(Q)} N^{*}(\alpha, Q, C) Q^{\left(15-12 \varepsilon_{1}-1 / 3\right)(\alpha-1)} \log N d \alpha .
\end{aligned}
$$

By the next-to-last inequality in Lemma 3.1, the last integral in (6.8) is

$$
\begin{aligned}
& \leq \int_{\eta(Q) \mathcal{L}}^{6} 15(167.67)(2)^{-1} \exp (3.116796 \lambda) Q^{\left(15-12 \varepsilon_{1}-1 / 3\right)(-\lambda / \mathcal{L})} d \lambda \\
& \leq 109\left(\frac{3 \omega}{1-\varepsilon_{3}}\right)^{3.849956} \leq 0.448 \omega^{3} .
\end{aligned}
$$


Combining all the above, we get for $\omega \leq 10^{-5}, \Sigma_{3} \leq 0.45 \omega^{3}$.

Comparison of Tables 1 and 2 in $\S 2$ shows that the bounds in Table 2 can be applied in all cases if $\widetilde{\beta}$ exists. And in view of the definition of $\omega$ in (5.12), one finds that the $\omega$ here plays the role of $\lambda_{1}$ in Table 2. Thus we can proceed as follows.

(ii) If $10^{-5}<\omega \leq 0.0025$, then for any zero $\varrho=\beta+i \gamma \neq \widetilde{\beta}$ of the function $(2.1)$ we have $\beta \leq 1-4.55 / \mathcal{L}$. Using the estimates for $C_{1}$ to $C_{4}$ in the case where $\widetilde{\beta}$ does not exist, by Lemma 3.1 , we get

$$
\begin{aligned}
\Sigma_{3} & \leq C_{1}+C_{2}+C_{3}+C_{4}+15 \int_{4.55}^{6} E\left(\varepsilon_{1}, \lambda\right) N_{5}^{*} d \lambda \\
& \leq C_{1}+C_{2}+C_{3}+C_{4}+7.1 \cdot 10^{-22} \leq 7.2 \cdot 10^{-22} \leq 10^{-6} \omega^{3} .
\end{aligned}
$$

(iii) If $0.0025<\omega \leq 0.066$, then for any zero $\varrho=\beta+i \gamma \neq \widetilde{\beta}$ of the function $(2.1)$ we have $\beta \leq 1-2 / \mathcal{L}$. Thus $\Sigma_{3} \leq C_{1}+C_{2}+C_{3}+C_{4}+C_{5} \leq$ $8.8 \cdot 10^{-9} \leq 0.5633 \omega^{3}$.

(iv) If $0.066<\omega \leq 0.2$, then $\beta \leq 1-1.16 / \mathcal{L}$. Thus

$$
\Sigma_{3} \leq \sum_{l=1}^{5} C_{l}+15 \int_{1.16}^{2} E\left(\varepsilon_{1}, \lambda\right) N_{6}^{*} d \lambda \leq 0.000142 \leq 0.5 \omega^{3} .
$$

(v) If $0.2<\omega \leq 0.306$, then $\beta \leq 1-0.867 / \mathcal{L}$. Hence

$$
\Sigma_{3} \leq \sum_{l=1}^{6} C_{l}+15 \int_{0.867}^{1} E\left(\varepsilon_{1}, \lambda\right) N_{7}^{*} d \lambda \leq 0.00375 \leq 0.5 \omega^{3} .
$$

(vi) If $0.306<\omega \leq 0.364$, then $\beta \leq 1-0.75 / \mathcal{L}$. Hence

$$
\Sigma_{3} \leq \sum_{l=1}^{6} C_{l}+15 \int_{0.75}^{1} E\left(\varepsilon_{1}, \lambda\right) N_{7}^{*} d \lambda \leq 0.0136 \leq 0.5 \omega^{3} .
$$

Combining all the estimates for $\Sigma_{3}$ from the above cases (i) to (vi), we conclude that when $\widetilde{\beta}$ does indeed exist,

$$
\Sigma_{3} \leq 0.5633 \omega^{3} \text {. }
$$

By (6.6) and (6.9), the proof of Lemma 6.2 is complete.

7. The estimation of $M_{2}$. We first give some preliminary lemmas.

Lemma 7.1. For any absolute constant $C \geq 1$, we have for $1 \leq j \leq 3$,

$$
\begin{aligned}
S_{2, j} & :=\sum_{q \leq Q} \sum_{\chi(\bmod q)}^{*} \sum_{C \leq|\gamma| \leq T}\left\{\int_{-\tau / q}^{\tau / q}\left|\int_{N_{j}^{\prime}}^{N_{j}} x^{\varrho-1} e\left(a_{j} x \eta\right) d x\right|^{3} d \eta\right\}^{1 / 3} \\
& \ll C^{-1 / 6}\left|a_{j}\right|^{-1} N^{2 / 3} .
\end{aligned}
$$


Proof. The integral with respect to $\eta$ in the above representation is

$$
\left|a_{j}\right|^{-3 \beta} \int_{-\tau / q}^{\tau / q}\left|\int_{N / 4}^{N} x^{\varrho-1} e(x \eta) d x\right|^{3} d \eta .
$$

Hence by Lemma 4.3 , the innermost sum $\sum_{C \leq|\gamma| \leq T}$ in the representation of $S_{2, j}$ is

$$
\begin{aligned}
& \ll \sum_{C \leq|\gamma| \leq T}\left|a_{j}\right|^{-\beta}\left\{\int_{\substack{\eta \in[0, \tau / q] \\
\eta \leq|\gamma| /(4 \pi N)}}\left(N^{\beta}|\gamma|^{-1}\right)^{3} d \eta\right. \\
& \left.+\int_{\substack{\eta \in[0, \tau / q] \\
|\gamma| /(4 \pi N)<\eta \leq 4|\gamma| /(\pi N)}}\left(N^{\beta}|\gamma|^{-1 / 2}\right)^{3} d \eta+\int_{\substack{\eta \in[0, \tau / q] \\
\eta>4|\gamma| /(\pi N)}}\left(N^{\beta-1} \eta^{-1}\right)^{3} d \eta\right\}^{1 / 3} \\
& \ll \sum_{C \leq|\gamma| \leq T}\left|a_{j}\right|^{-\beta} N^{\beta-1 / 3}|\gamma|^{-1 / 6} \ll C^{-1 / 6}\left|a_{j}\right|^{-1} N^{2 / 3} \sum_{|\gamma| \leq T} N_{j}^{\beta-1},
\end{aligned}
$$

by noting (4.2). Thus by Lemma 4.5 we get the desired result.

LEMMA 7.2. We have for $1 \leq j \leq 3$,

$$
\begin{aligned}
S_{3, j} & :=\sum_{q \leq Q} \sum_{\chi(\bmod q)}^{*} \sum_{|\gamma| \leq T}^{\prime}\left\{\int_{-\tau / q}^{\tau / q}\left|\int_{N_{j}^{\prime}}^{N_{j}} x^{\varrho-1} e\left(a_{j} x \eta\right) d x\right|^{3} d \eta\right\}^{1 / 3} \\
& \ll\left|a_{j}\right|^{-1} N^{2 / 3} .
\end{aligned}
$$

Proof. In view of Lemma 7.1, we only need to prove the lemma for $T=1$. This follows from Lemmas 4.3 and 4.5 .

Lemma 7.3. For the $\varepsilon_{2}>0$ given as in Lemma 6.1, let $Q>K\left(\varepsilon_{2}\right)$ which is a positive constant depending on $\varepsilon_{2}$ only. If $\widetilde{\beta}$ exists and satisfies $\omega \leq \varepsilon_{2}$, then $S_{3, j}$ defined as in Lemma 7.2 can be estimated further as $S_{3, j} \ll \varepsilon_{2}^{1 / 2} \omega^{3} N^{2 / 3}\left|a_{j}\right|^{-1}$.

Proof. We write

$$
\begin{aligned}
S_{3, j}= & \left\{\sum_{q \leq Q} \sum_{\chi(\bmod q)}^{*} \sum_{|\gamma| \leq 15 Q^{1+\varepsilon_{1}} q^{-1}}^{\prime}+\sum_{q \leq Q} \sum_{\chi(\bmod q)}^{*} \sum_{15 Q^{1+\varepsilon_{1}} q^{-1}<|\gamma| \leq T}^{\prime}\right\} \\
& \times\left\{\int_{-\tau / q}^{\tau / q}\left|\int_{N_{j}^{\prime}}^{N_{j}} x^{\varrho-1} e\left(a_{j} x \eta\right) d x\right|^{3} d \eta\right\}^{1 / 3} .
\end{aligned}
$$

By a similar argument to the proof of Lemma 7.1, the innermost sum $\sum_{|\gamma| \leq 15 Q^{1+\varepsilon_{1} q^{-1}}}^{\prime}$ in the first multi-sum in (7.2) is

$$
\ll\left|a_{j}\right|^{-1} N^{2 / 3} \sum_{|\gamma| \leq 15 Q^{1+\varepsilon_{1}} q^{-1}}^{\prime} N_{j}^{\beta-1} .
$$


Thus by Lemma 6.1 , the first multi-sum in $(7.2)$ is $\ll \varepsilon_{2}^{1 / 2} \omega^{3}\left|a_{j}\right|^{-1} N^{2 / 3}$ as desired. We now consider the second multi-sum in (7.2). In view of $|\eta| \leq \tau q^{-1}$ and $\tau=N^{-1} Q^{1+\varepsilon_{1}}$, we always have $|\eta| \leq|\gamma| /(4 \pi N)$. Thus by (7.1) and the second inequality in Lemma 4.3 , the innermost sum $\sum_{15 Q^{1+\varepsilon_{1} q^{-1}}<|\gamma| \leq T}^{\prime}$ in the second multi-sum in (7.2) can be estimated as

$$
\begin{aligned}
& \ll \sum_{15 Q^{1+\varepsilon_{1}} q^{-1}<|\gamma| \leq T}^{\prime}\left|a_{j}\right|^{-\beta}\left\{\int_{-\tau / q}^{\tau / q} N^{3 \beta}|\gamma|^{-3} d \eta\right\}^{1 / 3} \\
& \ll Q^{-2 \varepsilon_{1} / 3}\left|a_{j}\right|^{-1} N^{2 / 3} \sum_{15 Q^{1+\varepsilon_{1}} q^{-1}<|\gamma| \leq T}^{\prime} N_{j}^{\beta-1} .
\end{aligned}
$$

Thus by Lemma 4.5 and in view of (6.2), the second multi-sum in (7.2) can be bounded by $\varepsilon_{2}^{1 / 2} \omega^{3}\left|a_{j}\right|^{-1} N^{2 / 3}$ again. The proof of Lemma 7.3 is complete.

Lemma 7.4. Let $M_{0}$ be defined as in (5.10). If (i) all the $a_{j}$ 's are positive and $b=N$, or (ii) not all the $a_{j}$ 's are of the same sign and $N \geq 3|b|$, then

$$
M_{0} \gg N^{2}\left|a_{1} a_{2} a_{3}\right|^{-1} \prod_{p} s(p) .
$$

Proof. The lemma can be proved in precisely the same way as [LT1, Lemma 7.2].

Now we come to estimate $M_{2}$. We consider two cases according as the exceptional zero $\widetilde{\beta}$ exists or not.

(I) $\widetilde{\beta}$ exists. Recall from (5.1) that there are 19 terms in the integrand of $M_{2}$ and they are of the following 6 types:

(i) 3 terms of the form $\left\{\prod_{j=1}^{2} G\left(a_{j} h, q\right) I_{j}(\eta)\right\} \mathcal{G}_{3}(h, q, \eta)$;

(ii) 6 terms of the form $\delta(q) G\left(a_{1} h, q\right) I_{1}(\eta) G\left(a_{2} h, \widetilde{\chi} \chi_{0}\right) \widetilde{I}_{2}(\eta) \mathcal{G}_{3}(h, q, \eta)$;

(iii) 3 terms of the form $\delta(q)\left\{\prod_{j=1}^{2} G\left(a_{j} h, \widetilde{\chi} \chi_{0}\right) \widetilde{I}_{j}(\eta)\right\} \mathcal{G}_{3}(h, q, \eta)$;

(iv) 3 terms of the form $G\left(a_{1} h, q\right) I_{1}(\eta) \prod_{j=2}^{3} \mathcal{G}_{j}(h, q, \eta)$;

(v) 3 terms of the form $\delta(q) G\left(a_{1} h, \widetilde{\chi} \chi_{0}\right) \widetilde{I}_{1}(\eta) \prod_{j=2}^{3} \mathcal{G}_{j}(h, q, \eta)$;

(vi) the remaining term $\prod_{j=1}^{3} \mathcal{G}_{j}(h, q, \eta)$.

The treatment of these six types is quite similar. We illustrate the details with a term belonging to the fifth type, namely,

$$
\begin{aligned}
M_{25}:= & \sum_{q \leq Q} \varphi(q)^{-3} \sum_{h=1}^{q} e\left(-\frac{b}{q} h\right) \\
& \times \int_{-\tau / q}^{\tau / q} \delta(q) G\left(a_{1} h, \widetilde{\chi} \chi_{0}\right) \widetilde{I}_{1}(\eta) e(-b \eta) \prod_{j=2}^{3} \mathcal{G}_{j}(h, q, \eta) d \eta .
\end{aligned}
$$


In view of (4.9), (4.10) and (5.5), the above is

$$
\begin{aligned}
= & \sum_{\substack{q \leq Q \\
\widetilde{r} \mid q}} \varphi(q)^{-3} \sum_{h=1}^{q} e\left(-\frac{b}{q} h\right) G\left(a_{1} h, \widetilde{\chi} \chi_{0}\right) \\
& \times \sum_{\chi_{2}(\bmod q)} \sum_{\chi_{3}(\bmod q)}\left(\prod_{j=2}^{3} G\left(a_{j} h, \bar{\chi}_{j}\right)\right) \\
& \times \sum_{\left|\gamma_{2}\right| \leq T}^{\prime} \sum_{\left|\gamma_{3}\right| \leq T}^{\prime} \int_{-\tau / q}^{\tau / q} e(-b \eta) \widetilde{I}_{1}(\eta)\left(\prod_{j=2}^{3} \int_{N_{j}^{\prime}}^{N_{j}} x^{\varrho_{j}-1} e\left(a_{j} x \eta\right) d x\right) d \eta \\
= & \sum_{r_{2} \leq Q} \sum_{\chi_{2}\left(\bmod r_{2}\right)}^{*} \sum_{\left|\gamma_{2}\right| \leq T}^{\prime} \sum_{r_{3} \leq Q} \sum_{\chi_{3}\left(\bmod r_{3}\right)} \sum_{\left|\gamma_{3}\right| \leq T} \varphi(q)^{-3} \\
& \times Z\left(q ; \tilde{r}, r_{2}, r_{3}\right] \mid q \\
& \times\left(\prod_{j=2}^{3} \int_{N_{j}^{\prime}}^{N_{j}} x^{\varrho_{j}-1} e\left(\bar{\chi}_{2}, \bar{\chi}_{3}\right) \int_{-\tau / q}^{\tau / q} e(-b \eta) \widetilde{I}_{1}(\eta)\right.
\end{aligned}
$$

Noting that $\left[\widetilde{r}, r_{2}, r_{3}\right] \mid q$ implies $r_{2}, r_{3} \leq q$, and applying Cauchy's inequality, the absolute value of the last integral with respect to $\eta$ in (7.3) is

$$
\begin{aligned}
\leq & \left\{\int_{-\tau / q}^{\tau / q}\left|\int_{N_{1}^{\prime}}^{N_{1}} x^{\widetilde{\beta}-1} e\left(a_{1} x \eta\right) d x\right|^{3} d \eta\right\}^{1 / 3} \\
& \times \prod_{j=2}^{3}\left\{\int_{-\tau / r_{j}}^{\tau / r_{j}}\left|\int_{N_{j}^{\prime}}^{N_{j}} x^{\varrho_{j}-1} e\left(a_{j} x \eta\right) d x\right|^{3} d \eta\right\}^{1 / 3} .
\end{aligned}
$$

By Lemma 4.3, the first term in the above product can be estimated easily as

$$
\ll\left|a_{1}\right|^{-1} N^{2 / 3} \text {. }
$$

Now we argue according to whether $\omega \leq \varepsilon_{2}$ or not. If $\omega \leq \varepsilon_{2}$, we substitute (7.5) into (7.4), and then into (7.3). Then using (5.8) to estimate the sum over $q$ in (7.3), and applying Lemma 7.3, (7.3) can be estimated further as

$$
\ll \varepsilon_{2} N^{2}\left|a_{1} a_{2} a_{3}\right|^{-1} \omega^{6} \prod_{p} s(p) .
$$

If $\omega>\varepsilon_{2}$, we write (7.3) further as 


$$
\begin{aligned}
& =\left\{\sum_{r_{2} \leq Q} \sum_{\chi_{2}}^{*} \sum_{\left(\bmod r_{2}\right)}^{\prime} \sum_{\left|\gamma_{2}\right| \leq C} \sum_{r_{3} \leq Q}^{*} \sum_{\chi_{3}\left(\bmod r_{3}\right)}^{\prime}\right. \\
& \left.+\sum_{r_{2} \leq Q} \sum_{\chi_{2}\left(\bmod r_{2}\right)}^{*} \sum_{\left|\gamma_{2}\right| \leq T}^{\prime} \sum_{r_{3} \leq Q} \sum_{\chi_{3}\left(\bmod r_{3}\right)}^{*} \sum_{\left|\gamma_{3}\right| \leq T}^{\prime}\right\} \\
& \text { at least one of }\left|\gamma_{j}\right| \geq C, j=2,3 \\
& \times \sum_{\substack{q \leq Q \\
\left[\widetilde{r}, r_{2}, r_{3}\right] \mid q}} \varphi(q)^{-3} Z\left(q ; \tilde{\chi}, \bar{\chi}_{2}, \bar{\chi}_{3}\right) \\
& \times \int_{-\tau / q}^{\tau / q} e(-b \eta) \widetilde{I}_{1}(\eta)\left(\prod_{j=2}^{3} \int_{N_{j}^{\prime}}^{N_{j}} x^{\varrho_{j}-1} e\left(a_{j} x \eta\right) d x\right) d \eta .
\end{aligned}
$$

For the second multi-sum inside the curly brackets in (7.7), similar to the above arguments, we substitute (7.5) into (7.4), and then into (7.7). Then using (5.8) to estimate the sum over $q$, and applying Lemmas 7.1 and 7.2, the second multi-sum can be estimated as

$$
\ll N^{2}\left|a_{1} a_{2} a_{3}\right|^{-1} C^{-1 / 6} \prod_{p} s(p) .
$$

For the first multi-sum inside the curly brackets in (7.7), we first extend the range of the integration with respect to $\eta$ to $(-\infty, \infty)$ and let $R_{25}$ be the total error induced in (7.7) because of the extension of range. Then

$$
\begin{aligned}
R_{25} \ll & \sum_{r_{2} \leq Q} \sum_{\chi_{2}\left(\bmod r_{2}\right)}^{*} \sum_{\left|\gamma_{2}\right| \leq C}^{\prime} \sum_{r_{3} \leq Q} \sum_{\chi_{3}\left(\bmod r_{3}\right)}^{*} \sum_{\left|\gamma_{3}\right| \leq C}^{\prime} \sum_{\substack{q \leq Q \\
\left[\widetilde{r}, r_{2}, r_{3}\right] \mid q}} \varphi(q)^{-3} \\
& \times\left|Z\left(q ; \widetilde{\chi}, \bar{\chi}_{2}, \bar{\chi}_{3}\right)\right| \mid \int_{\tau / q}^{\infty}\left(\int_{N_{1}^{\prime}}^{N_{1}} x^{\widetilde{\beta}-1} e\left(a_{1} x \eta\right) d x\right) \\
& \times\left(\prod_{j=2}^{3} \int_{N_{j}^{\prime}}^{N_{j}} x^{\varrho_{j}-1} e\left(a_{j} x \eta\right) d x\right) e(-b \eta) d \eta \mid
\end{aligned}
$$

By Cauchy's inequality, the absolute value of the integral with respect to $\eta$ in $(7.9)$ is

$$
\begin{aligned}
\leq & \left\{\int_{\tau / q}^{\infty}\left|\int_{N_{1}^{\prime}}^{N_{1}} x^{\widetilde{\beta}-1} e\left(a_{1} x \eta\right) d x\right|^{3} d \eta\right\}^{1 / 3} \\
& \times \prod_{j=2}^{3}\left\{\int_{\tau / q}^{\infty}\left|\int_{N_{j}^{\prime}}^{N_{j}} x^{\varrho_{j}-1} e\left(a_{j} x \eta\right) d x\right|^{3} d \eta\right\}^{1 / 3} .
\end{aligned}
$$


For any $\varrho=\beta+i \gamma$ with $|\gamma| \leq C$, we have for $1 \leq j \leq 3$,

$$
\int_{\tau / q}^{\infty}\left|\int_{N_{j}^{\prime}}^{N_{j}} x^{\varrho-1} e\left(a_{j} x \eta\right) d x\right|^{3} d \eta=\left|a_{j}\right|^{-3 \beta} \int_{\tau / q}^{\infty}\left|\int_{N / 4}^{N} x^{\varrho-1} e(x \eta) d x\right|^{3} d \eta .
$$

Since $\tau / q=Q^{1+\varepsilon} /(q N) \geq Q^{\varepsilon} N^{-1}>4 C /(\pi N)$, by the last inequality in Lemma 4.3 , the above can be estimated further as

$$
\ll\left|a_{j}\right|^{-3 \beta} \int_{\tau / q}^{\infty}\left(N^{\beta-1} \eta^{-1}\right)^{3} d \eta \ll N^{2} Q^{-2 \varepsilon_{1}}\left|a_{j}\right|^{-3} N_{j}^{3(\beta-1)} .
$$

Hence (7.10) is

$$
\ll N^{2} Q^{-2 \varepsilon_{1}}\left|a_{1} a_{2} a_{3}\right|^{-1} N_{1}^{\widetilde{\beta}-1} N_{2}^{\beta_{2}-1} N_{3}^{\beta_{3}-1} .
$$

Now, substitute the bound (7.11) into (7.9), and then use (5.8) to estimate the sum over $q$ in (7.9). Then by Lemma 6.2 we get

$$
R_{25} \ll N^{2} Q^{-2 \varepsilon_{1}}\left|a_{1} a_{2} a_{3}\right|^{-1} \prod_{p} s(p) .
$$

Therefore by (7.7) and (7.8) and noting $Q^{-2 \varepsilon_{1}} \leq C^{-1 / 6}$, we can summarize that

$$
\begin{aligned}
M_{25}= & \sum_{r_{2} \leq Q} \sum_{\chi_{2}\left(\bmod r_{2}\right)}^{*} \sum_{\left|\gamma_{2}\right| \leq C}^{\prime} \sum_{r_{3} \leq Q} \sum_{\chi_{3}\left(\bmod r_{3}\right)}^{*} \sum_{\left|\gamma_{3}\right| \leq C}^{\prime} \sum_{\substack{q \leq Q \\
\left[\widetilde{r}, r_{2}, r_{3}\right] \mid q}} \varphi(q)^{-3} \\
& \times Z\left(q ; \tilde{\chi}, \bar{\chi}_{2}, \bar{\chi}_{3}\right) \int_{-\infty}^{\infty} e(-b \eta)\left(\int_{N_{1}^{\prime}}^{N_{1}} x^{\widetilde{\beta}-1} e\left(a_{1} x \eta\right) d x\right) \\
& \times\left(\prod_{j=2}^{3} \int_{N_{j}^{\prime}}^{N_{j}} x^{\varrho_{j}-1} e\left(a_{j} x \eta\right) d x\right) d \eta+O\left(N^{2}\left|a_{1} a_{2} a_{3}\right|^{-1} C^{-1 / 6} \prod_{p} s(p)\right) .
\end{aligned}
$$

Note that by (4.2) and (5.6) we have, for $\left(x_{1}, x_{2}\right) \in \mathcal{D}$ and $j=1,2,3$, $N x_{j} \geq N_{j}^{\prime}$. Hence using Lemmas 5.1, 5.2, 6.2, (5.10) and $\left(N x_{1}\right)^{\widetilde{\beta}-1} \leq 1$, we get, when $\widetilde{\beta}$ exists and satisfies $\omega>\varepsilon_{2}$,

$$
\begin{aligned}
\left|M_{25}\right| \leq & N^{2}\left|a_{3}\right|^{-1}(2.140782)\left(0.5633 \omega^{3}\right)^{2}\left(\prod_{p} s(p)\right) \int_{\mathcal{D}} d x_{1} d x_{2} \\
& +O\left(N^{2}\left|a_{1} a_{2} a_{3}\right|^{-1} C^{-1 / 6} \prod_{p} s(p)\right) \\
\leq & (2.140782)\left(0.5633 \omega^{3}\right)^{2} M_{0}+O\left(N^{2}\left|a_{1} a_{2} a_{3}\right|^{-1} C^{-1 / 6} \prod_{p} s(p)\right) .
\end{aligned}
$$


If $C \geq \varepsilon_{2}^{-37}$, then by Lemma 7.4 , the above $O$-term can be absorbed and hence under the assumptions in Lemma 7.4,

$$
\left|M_{25}\right| \leq\left(2.140782+\varepsilon_{4}\left(\varepsilon_{2}\right)\right)\left(0.5633 \omega^{3}\right)^{2} M_{0},
$$

where $\varepsilon_{4}\left(\varepsilon_{2}\right)$ is a positive number depending on $\varepsilon_{2}$ only. And $\varepsilon_{4}$ becomes sufficiently small if $\varepsilon_{2}$ is chosen to be sufficiently small. The comparison of (7.6) and (7.12) shows that under the conditions in Lemma 7.4, the upper bound (7.12) for $M_{25}$ always holds whenever $\widetilde{\beta}$ exists. In precisely the same way we can derive that under the conditions of Lemma 7.4 and $C \geq \varepsilon_{2}^{-55}$,

$$
\begin{aligned}
& \left|M_{21}\right|,\left|M_{22}\right|,\left|M_{23}\right| \leq\left(2.140782+\varepsilon_{4}\left(\varepsilon_{2}\right)\right)\left(0.5633 \omega^{3}\right) M_{0}, \\
& \left|M_{24}\right| \leq\left(2.140782+\varepsilon_{4}\left(\varepsilon_{2}\right)\right)\left(0.5633 \omega^{3}\right)^{2} M_{0} \\
& \left|M_{26}\right| \leq\left(2.140782+\varepsilon_{4}\left(\varepsilon_{2}\right)\right)\left(0.5633 \omega^{3}\right)^{3} M_{0} .
\end{aligned}
$$

Consequently we obtain, under the assumptions of Lemma 7.4 and $C \geq$ $\varepsilon_{2}^{-55}$

$$
\begin{aligned}
M_{2} \leq & \left(2.140782+\varepsilon_{4}\left(\varepsilon_{2}\right)\right) \\
& \times\left(12\left(0.5633 \omega^{3}\right)+6\left(0.5633 \omega^{3}\right)^{2}+\left(0.5633 \omega^{3}\right)^{3}\right) M_{0} \\
\leq & 15 \omega^{3} M_{0} .
\end{aligned}
$$

(II) $\widetilde{\beta}$ does not exist. In this case, there are only 7 terms in the integrand of $M_{2}$ and they are of the first, fourth and sixth type in the previous case (I). Treatment of these terms is completely the same as in case (I). With the use of the first inequality for $\Sigma_{3}$ in Lemma 6.2 instead of the second one, we obtain, under the assumptions of Lemma 7.4,

$$
\begin{aligned}
& \left|M_{21}\right| \leq(2.140782+\varepsilon)(0.096) M_{0}, \\
& \left|M_{24}\right| \leq(2.140782+\varepsilon)(0.096)^{2} M_{0}, \\
& \left|M_{26}\right| \leq(2.140782+\varepsilon)(0.096)^{3} M_{0},
\end{aligned}
$$

providing that the above $C$ is large enough. Consequently,

$$
M_{2} \leq(2.140782+\varepsilon)\left(3(0.096)+3(0.096)^{2}+(0.096)^{3}\right) M_{0} \leq 0.68 M_{0} .
$$

From (7.13) and (7.14) one can obtain

Lemma 7.5. For any $\varepsilon_{2}>0$ given as in Lemma 6.1 , let $Q>K\left(\varepsilon_{2}\right)$ which is a positive constant depending on $\varepsilon_{2}$ only. Under the assumptions of Lemma 7.4, we have

$$
M_{2} \leq \begin{cases}0.68 M_{0} & \text { if } \widetilde{\beta} \text { does not exist } \\ 15 \omega^{3} M_{0} & \text { if } \widetilde{\beta} \text { exists. }\end{cases}
$$

REMARK 3. For a positive lower bound for $I(b)$, by (5.2), Lemma 5.3 and Lemma 8.1 below, it is sufficient to have $M_{2} \leq(1-\varepsilon) M_{0}$ when $\widetilde{\beta}$ does not 
exist since then $M_{3}=0$. By the proof of Lemma 7.5 and the first inequality for $\Sigma_{3}$ in Lemma 6.2, the numerical upper bound for $M_{2}$ is increasing with respect to the $\theta$ in (4.1). Hence, in view of the above upper bound $0.68 M_{0}$ for $M_{2}$ in (7.14), one can expect that a larger $\theta>1 /\left(15-11 \varepsilon_{1}\right)$ is permitted. But numerical experiments show that, by the present method, $\theta=1 / 14$ fails for the above purpose. Thus, to make the statements clearer, we take $\theta=1 /\left(15-11 \varepsilon_{1}\right)$.

\section{Proof of Theorem 1}

Lemma 8.1. Let $I_{2}(b)$ be defined as in (4.7). Then

$$
I_{2}(b) \ll N^{2} Q^{-1 / 2}\left|a_{1} a_{2} a_{3}\right|^{-1 / 2} L^{5} .
$$

Proof. The lemma can be proved in precisely the same way as [LT1, Lemma 7.1] with the coefficients $a_{j}$ taken into consideration.

Lemma 8.2. Let $I_{1}(b)$ be defined as in (4.7) and $\theta$ be given as in (4.1). Under the assumptions of Lemma 7.4 and (4.4), we have $I_{1}(b) \gg \omega^{3} M_{0}$.

Proof. If the exceptional zero $\widetilde{\beta}$ does not exist, then there is no $M_{3}$ in (5.2). Thus by (5.2), (5.9) and Lemma 7.5, and then by (4.16) and (5.11) we get

$$
\begin{aligned}
I_{1}(b) & \geq(1-0.68) M_{0}+O\left(\Omega_{1}+\Omega_{2}\right) \\
& \gg M_{0}+O\left(N^{2} Q^{2.5+\varepsilon_{1}}\left|a_{1} a_{2} a_{3}\right|^{-1} T^{-1} L^{2}+N^{2} Q^{-1+\varepsilon_{1} / 4}\left|a_{1} a_{2} a_{3}\right|^{-1}\right) .
\end{aligned}
$$

In view of (5.7), Lemma 7.4 and $T=Q^{3}$ (in (4.1)), the above $O$-term can be absorbed into $M_{0}$ and hence $I_{1}(b) \gg M_{0}$. If $\widetilde{\beta}$ exists and the corresponding $\widetilde{r} \leq Q^{1-\varepsilon_{1} / 3}$, then by (5.2), Lemma 5.5 and Lemma 7.5 , and hence by (4.16) and (5.11) we get

$$
\begin{aligned}
I_{1}(b) \geq & 5 \omega^{3} M_{0}+O\left(\Omega_{1}+\widetilde{r} \Omega_{2}+N^{2} Q^{-1-\varepsilon_{1}}\right) \\
\gg & \omega^{3} M_{0}+O\left(N^{2} Q^{2.5+\varepsilon_{1}}\left|a_{1} a_{2} a_{3}\right|^{-1} T^{-1} L^{2}\right. \\
& \left.+Q^{1-\varepsilon_{1} / 3} N^{2} Q^{-1+\varepsilon_{1} / 4}\left|a_{1} a_{2} a_{3}\right|^{-1}+N^{2} Q^{-1-\varepsilon_{1}}\right) .
\end{aligned}
$$

In view of (5.7), (6.2), Lemma 7.4 and $T=Q^{3}$, the above $O$-term can be absorbed and then $I_{1}(b) \gg \omega^{3} M_{0}$, providing that $Q \gg A^{3+2 \varepsilon_{1}}$. If $\widetilde{\beta}$ exists and the corresponding $\widetilde{r}>Q^{1-\varepsilon_{1} / 3}$, then by the same arguments as above except that now we use Lemma 5.6 instead of Lemma 5.5, we get

$$
\begin{aligned}
I_{1}(b) \geq & \left(1-15 \omega^{3}\right) M_{0}+O\left(\Omega_{1}+\Omega_{2}+N^{2} \widetilde{r}^{-1} \log ^{3} L+N^{2} Q^{-1-\varepsilon_{1}}\right) \\
\gg & M_{0}+O\left(N^{2} Q^{2.5+\varepsilon_{1}}\left|a_{1} a_{2} a_{3}\right|^{-1} T^{-1} L^{2}\right. \\
& \left.+N^{2} Q^{-1+\varepsilon_{1} / 3} \log ^{3} L+N^{2} Q^{-1-\varepsilon_{1}}\right) \\
\gg & M_{0},
\end{aligned}
$$


providing that $Q$ is sufficiently large, specifically $Q \gg A^{3+2 \varepsilon_{1}}$. The proof of Lemma 8.2 is complete.

Proof of Theorem 1. By (4.7), Lemmas 8.1, 8.2 and 7.4 and in view of (6.2), we get

$$
I(b)=I_{1}(b)+I_{2}(b) \gg \omega^{3} N^{2}\left|a_{1} a_{2} a_{3}\right|^{-1}
$$

providing that $Q$ is sufficiently large, specifically $Q \gg A^{3+2 \varepsilon_{1}}$, that is, $N \gg A^{\left(3+2 \varepsilon_{1}\right)\left(15-11 \varepsilon_{1}\right)}=A^{45-3 \varepsilon_{1}-22 \varepsilon_{1}^{2}}$ with $\varepsilon_{1}$ small enough. By the assumptions of Lemma 7.4, the proof of Theorem 1 (and of the conclusion given in Remark 2) is complete.

Acknowledgements. The research is partially supported by Hong Kong RGC Research Grant (No. HKU518/96P).

\section{References}

[B] A. Baker, On some diophantine inequalities involving primes, J. Reine Angew. Math. 228 (1967), 166-181.

[Che] J. R. Chen, On the least prime in an arithmetical progression and theorems concerning the zeros of Dirichlet's L-functions (II), Sci. Sinica 22 (1979), 859889.

[Cho] K. K. S. Choi, A numerical bound for Baker's constant - some explicit estimates for small prime solutions of linear equations, Bull. Hong Kong Math. Soc. 1 (1997), 1-19.

[CLT] K. K. Choi, M. C. Liu and K. M. Tsang, Conditional bounds for small prime solutions of linear equations, Manuscripta Math. 74 (1992), 321-340.

[D] H. Davenport, Multiplicative Number Theory, 2nd ed., Grad. Texts in Math. 74, Springer, 1980.

[G1] S. Graham, Applications of sieve methods, Ph.D. thesis, University of Michigan, 1977.

[G2] -, On Linnik's constant, Acta Arith. 39 (1981), 163-179.

[H-B] D. R. Heath-Brown, Zero-free regions for Dirichlet L-functions, and the least prime in an arithmetic progression, Proc. London Math. Soc. 64 (1992), 265-338.

[J] M. Jutila, On Linnik's constant, Math. Scand. 41 (1977), 45-62.

[L] Yu. V. Linnik, On the least prime in an arithmetic progression (I, II), Rec. Math. (Mat. Sb.) N. S. 15 (57) (1944), 139-178; 347-368.

[LLW] J. Y. Liu, M. C. Liu and T. Z. Wang, The number of powers of 2 in a representation of large even integers (II), Sci. China, to appear.

[Li1] M. C. Liu, A bound for prime solutions of some ternary equations, Math. Z. 188 (1985), 313-323.

[Li2] - An improved bound for prime solutions of some ternary equations, ibid. 194 (1987), 573-583.

[LT1] M. C. Liu and K. M. Tsang, Small prime solutions of linear equations, in: Théorie des Nombres, J.-M. De Koninck and C. Levesque (eds.), de Gruyter, Berlin, 1989, 595-624.

[LT2] - - - Recent progress on a problem of A. Baker, in: Séminaire de Théorie des Nombres, Paris 1991-1992, Progr. Math. 116, Birkhäuser, 1993, 121-133. 
[PP] C. D. Pan and C. B. P an, Goldbach Conjecture (English version), Science Press, Beijing, 1992.

Department of Mathematics

The University of Hong Kong

Pokfulam Road, Hong Kong

E-mail: matmcliu@hkucc.hku.hk
Department of Mathematics

Henan University

Kaifeng, Henan 475001

P.R. China

Received on 22.2.1998

and in revised form on 22.5.1998 